\title{
Coaxial two-channel high-gradient dielectric wakefield accelerator
}

\author{
G. V. Sotnikov, ${ }^{1,2, *}$ T. C. Marshall, ${ }^{3,2, \dagger}$ and J. L. Hirshfield ${ }^{4,2, \dagger}$ \\ ${ }^{1}$ NSC Kharkov Institute of Physics and Technology, 1 Akademicheskaya St., Kharkov 61108, Ukraine \\ ${ }^{2}$ Omega-P, Inc., 199 Whitney Avenue, New Haven, Connecticut 06511, USA \\ ${ }^{3}$ Columbia University, New York City, New York 10027, USA \\ ${ }^{4}$ Yale University, New Haven, Connecticut 06520, USA \\ (Received 27 March 2009; published 22 June 2009)
}

\begin{abstract}
A new scheme for a dielectric wakefield accelerator is proposed that employs a cylindrical multizone dielectric structure configured as two concentric dielectric tubes with outer and inner vacuum channels for drive and accelerated bunches. Analytical and numerical studies have been carried out for such coaxial dielectric-loaded structures (CDS) for high-gradient acceleration. An analytical theory of wakefield excitation by particle bunches in a multizone CDS has been formulated. Numerical calculations are presented for an example of a CDS using dielectric tubes with dielectric permittivity 5.7, having external diameters of 2.121 and $0.179 \mathrm{~mm}$ with inner diameters of 2.095 and $0.1 \mathrm{~mm}$. An annular $5 \mathrm{GeV}, 6 \mathrm{nC}$ electron bunch with rms length of $0.035 \mathrm{~mm}$ energizes a wakefield on the structure axis having an accelerating gradient of $\sim 600 \mathrm{MeV} / \mathrm{m}$ with a transformer ratio $\sim 8: 1$. The period of the accelerating field is $\sim 0.33 \mathrm{~mm}$. If the width of the drive bunch channel is decreased, it is possible to obtain an accelerating gradient of $>1 \mathrm{GeV} / \mathrm{m}$ while keeping the transformer ratio approximately the same. Full numerical simulations using a particle-in-cell code have confirmed results of the linear theory and furthermore have shown the important influence of the quenching wave that restricts the region of the wakefield to within several periods following the drive bunch. Numerical simulations for another example have shown nearly stable transport of drive and accelerated bunches through the CDS, using a short train of drive bunches.
\end{abstract}

DOI: 10.1103/PhysRevSTAB.12.061302

\section{INTRODUCTION}

In this paper we describe a coaxial cylindrical twochannel dielectric wakefield traveling-wave accelerator (DWFA) that is energized by a drive bunch having an annular cross section. This configuration can have a number of unique attributes, including (a) a transformer ratio $T$, defined as the peak accelerating field that can be applied to a test particle divided by the average decelerating field experienced by the drive bunch, which can be considerably greater than the value of about 2 that is typical for a collinear DWFA; (b) design flexibility in the tradeoff between high $T$ and high acceleration gradient $G$; (c) fabrication of the accelerator structure which can be comparatively simple and precise; (d) continuous energy coupling from drive to test bunch in the central accelerator channel without need for transfer structures; (e) singlebunch operation with high values of $G$ that should minimize disruptions due to long-range wakefields; (f) inherent transverse focusing forces for particles in the accelerated bunch; and (g) nearly stable motion of the annular drive bunch.

It will be shown below that a mm-scale version of this class of structures can be designed with $T \gg 1$ that can be capable of developing accelerating wakefields $G>$

\footnotetext{
*sotnikov@kipt.kharkov.ua

tcm2@columbia.edu

زjay.hirshfield@yale.edu
}

PACS numbers: 41.75.Jv, 41.75.Lx, 41.75.Ht, 96.50.Pw

$1 \mathrm{GeV} / \mathrm{m}$ when excited by a single drive bunch with charge of a few $\mathrm{nC}$. This is naturally of great significance because of the high magnitude of $G$. But it is also significant because it is achieved with only a single drive bunch. Several years ago, a theory was published predicting that exceptionally high accelerating fields could be achieved in a collinear dielectric-loaded structure by superimposing wakefields from successive bunches in a periodic train, so long as the bunch frequency matched the frequency of the dominant luminous wakefield mode $[1,2]$. Since then, that theory has been shown to be incomplete, since quenching fields that originate at the input boundary of the structure can interfere with the longer-range components of the wakefields, destroying their periodicity and greatly diminishing their magnitude [3,4]. Quenching fields are not included in the theory presented in Ref. [1]. Thus, we are now led to conclude that multibunch drive schemes with more than a few bunches are probably ineffective, and that single-bunch operation for a DWFA appears to be the most promising choice for achieving accelerating gradients of 100 's of $\mathrm{MeV} / \mathrm{m}$. Accordingly, the coaxial DWFA structure described here is being studied and developed with a long-range view towards its possible application in a future high-gradient, multi-TeV electron-positron collider operating in a single-bunch mode. Nevertheless, an example of multibunch wakefield excitation is presented where interference from quenching waves is not overwhelming. The limited conditions under which this can occur will be discussed. 
In conventional linear accelerators, the rf power used to accelerate the beam is derived from high-power rf amplifiers. To achieve multi-TeV energies, high accelerating gradients are necessary to limit the lengths of the two linacs, but the number of amplifiers needed could become excessive. Thus, alternative two-beam schemes have been proposed [5] wherein rf power is extracted from a lowenergy, high current drive beam which is decelerated in power extraction and transfer structures. This power is then fed into the structures of the main linac, and used to accelerate the high-energy, low-current main beam. The most active two-beam project now under study is the Compact Linear Collider (CLIC) concept at CERN [6], where an rf frequency of $12 \mathrm{GHz}$ has been chosen for the fields in the acceleration channel, in the expectation of sustaining a working gradient of $100 \mathrm{MeV} / \mathrm{m}$ in a metallic structure with highly infrequent breakdowns.

In contrast to CLIC, the rf generation mechanism for the structure proposed here is by creation of wakefields (Cherenkov radiation) induced by passage of a charge bunch along a dielectric-lined drive channel. This radiation couples continuously into a parallel acceleration channel, without need for auxiliary coupling elements. A diagram for the cross section of the simplest coaxial structure in this class is shown in Fig. 1. The path of the drive bunch moving in the outer annular channel in Fig. 1 is separated from that of the accelerated bunch that moves in the central channel. A rectangular two-channel DWFA is now under construction for tests at the Argonne National Laboratory (ANL) - the Argonne Wakefield Accelerator (AWA) facil-

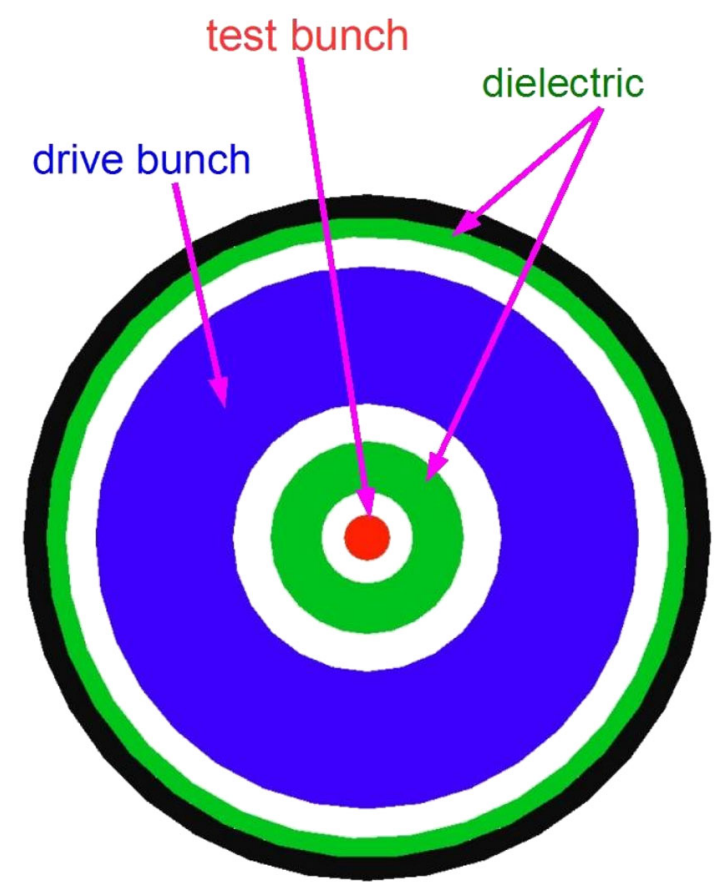

FIG. 1. (Color) Cross section of a coaxial DWFA. Dielectric tubes are green, drive bunch is blue; test bunch is red, and black outer shell is metal. ity. In another configuration, parallel cylindrical dielectric channels were used with a step-up coupling element between channels [7]; a prototype of that design was tested at the ANL-AWA facility [8].

The two-channel coaxial wakefield accelerator structure that will be investigated below is one version within a class of multilayered dielectric-loaded accelerating structures in which the central region and one of the concentric layers are vacuum channels [9-11]. Use of multilayered concentric dielectrics in a collinear acceleration scheme allows concentration of the excited wakefields in the central vacuum channel for particle acceleration. As a consequence, it is possible to reduce the high-frequency losses on metal walls of a surrounding waveguide [9,11], or to discard the surrounding waveguide altogether as in the design of the optical Bragg accelerator [10].

It is expected that a high acceleration field $(\sim 1-$ $2 \mathrm{GeV} / \mathrm{m}$ ) can be sustained in traveling-wave dielectric structures without breakdown because the dielectric is exposed to intense fields for only short times, according to supporting experimental evidence [12]. The breakdown limit of a wakefield structure is not determined by the slow filling time of a resonant cavity by electromagnetic energy, but rather by the much shorter time of the passing field pulses set up by the short bunches which generate the wakefield. The vacuum-based wakefield structure is geometrically rigid and therefore could be a precisely reproducible element in a staged system. Also, the structure is capable of accurate microfabrication, an important consideration when staging a large number of mm-scale modules that must be identical. The two-channel wakefield accelerator is already a step forward in improving the performance and versatility of wakefield accelerators by avoiding the requirement of collinearity between the drive bunch train and the accelerated bunch which occurs in a singlechannel DWFA device. The continuous coaxial dielectric configuration preserves the symmetry of the structure, which might result in fewer beam transport difficulties and provide a less complicated way to inject power into the accelerated bunch line than does the point-to-point power injection via transfer couplers as is required with conventional accelerator structures [7]. Finally, operation in a single-bunch mode should avoid beam disruptions that can arise due to long-range wakes acting on following bunches. These exceptional advantages for this accelerator structure seem to justify continued analysis, leading to design of a proof-of-principle experiment. This paper is intended as a step towards that goal.

\section{DISPERSION EQUATION AND EIGENMODES}

The coaxial two-channel dielectric wakefield accelerating structure analyzed analytically and numerically in the following sections is a particular case of a general multizone dielectric structure. One of the basic analytical methods for finding wakefields excited by charged particle 


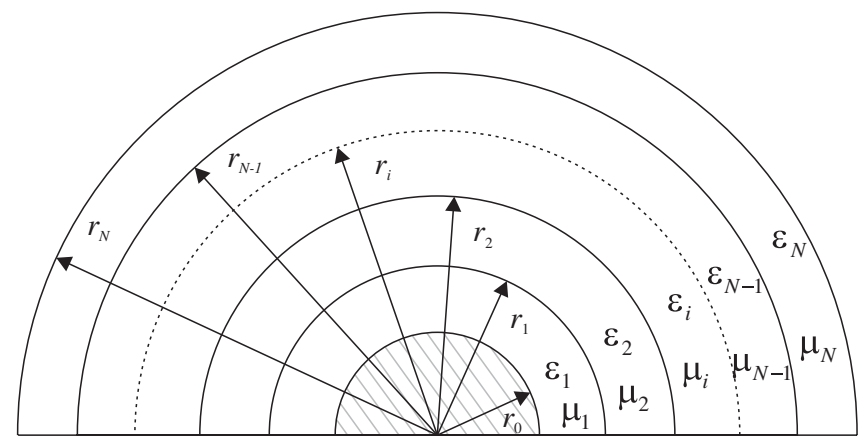

FIG. 2. Cross section of an $N$-zone dielectric-lined coaxial waveguide structure. The innermost vacuum zone of radius $r_{o}$ is for transport of the accelerated beam.

bunches in such structures is decomposition into eigenmodes, where the eigenfrequency and wave number for each mode correspond to a phase velocity equal to the drive bunch axial velocity. In this section we will derive the general dispersion equation of a multizone dielectric structure. The equations we obtain will be valid for any number of axisymmetric dielectric layers, each having its own scalar value of dielectric permittivity.

The sketch of a generalized multizone dielectric structure is shown in Fig. 2. The external radius of the first layer is $r_{1}$, and its internal radius is $r_{0}$. In general, the structure is composed of a simply connected zone (for the case $r_{0}=$ 0 ), and several doubly connected zones. The external layer of the structure $\left(r_{N-1}<r \leq r_{N}\right)$ can be bounded by free space or by a conducting metal pipe. Zones in which the drive bunch and the accelerated test particle reside are vacuum, wherein $\mu=\varepsilon=1$.

For deriving a compact form of the dispersion equation for a multizone dielectric structure, a matrix approach with use of corresponding transition matrices has proven effective. Recently this method has been applied for rectangular multizone dielectric structures [1]. The essence of this approach consists in knowing electromagnetic fields at one boundary of a zone, from which it is possible to find fields at the other boundary of the same zone, and then carrying this forward to include all zones [13]. We apply the method developed in Ref. [1] to obtain the dispersion equation of an axially symmetrical multizone cylindrical dielectric structure.

As trial solutions of Maxwell equations for components of the electromagnetic fields in each layer of the dielectric structure, we take the following:

$$
\begin{aligned}
& E_{z}^{(i)}=e_{z}^{(i)}(r) \exp \left[j\left(k_{z} z-\omega t\right)\right] \\
& E_{r}^{(i)}=j e_{r}^{(i)}(r) \exp \left[j\left(k_{z} z-\omega t\right)\right] \\
& H_{\varphi}^{(i)}=h_{\varphi}^{(i)}(r) \exp \left[j\left(k_{z} z-\omega t\right)\right],
\end{aligned}
$$

where $k_{z}$ and $\omega$ are the longitudinal wave number and frequency of a wave and $i$ is the zone number $(1 \leq i \leq$
$N)$. The absence of other components is valid when the source function (drive beam) is axisymmetric. The functions describing the transverse structure of the fields in the absence of external sources are obtained:

$$
e_{r}^{(i)}=\frac{k_{z}}{k^{2} \varepsilon_{i} \mu_{i}-k_{z}^{2}} \frac{\partial e_{z}^{(i)}}{\partial r}, \quad h_{\varphi}^{(i)}=\frac{k \varepsilon_{i}}{k_{z}} e_{r}^{(i)}
$$

Taking into account Eqs. (1) and (2), the solutions of the Maxwell equations for the $i$ th layer of a multizone dielectric structure have the form

$$
\begin{aligned}
& e_{z}^{(i)}=A^{(i)} J_{0}\left(\kappa_{i} r\right)+B^{(i)} Y_{0}\left(\kappa_{i} r\right), \\
& e_{r}^{(i)}=-A^{(i)} \frac{k_{z}}{\kappa_{i}} J_{1}\left(\kappa_{i} r\right)-B^{(i)} \frac{k_{z}}{\kappa_{i}} Y_{1}\left(\kappa_{i} r\right),
\end{aligned}
$$

where $\kappa_{i}^{2}=k^{2} \varepsilon_{i} \mu_{i}-k_{z}^{2}, J_{n}$, and $Y_{n}$ are Bessel functions and Weber functions of the $n$th order with $n=0,1$; and $A^{(i)}$ and $B^{(i)}$ are arbitrary constants which are defined from the boundary conditions. The boundary conditions comprise continuity of the longitudinal electric field $e_{z}^{(i)}$ and the transverse component of the displacement vector $\varepsilon_{i} e_{r}^{(i)}$. Taking this into account, the expressions can be rewritten in the matrix representation,

$$
\left(\begin{array}{c}
\frac{\varepsilon_{i}}{\kappa_{i}^{2}} \frac{\partial e_{z}^{(i)}(r)}{\partial r} \\
e_{z}^{(i)}(r)
\end{array}\right)=M_{i}(r)\left(\begin{array}{c}
A^{(i)} \\
B^{(i)}
\end{array}\right)
$$

where

$$
M_{i}(r)=\left(\begin{array}{cc}
-\frac{\varepsilon_{i}}{\kappa_{i}} J_{1}\left(\kappa_{i} r\right) & -\frac{\varepsilon_{i}}{\kappa_{i}} Y_{1}\left(\kappa_{i} r\right) \\
J_{0}\left(\kappa_{i} r\right) & Y_{0}\left(\kappa_{i} r\right)
\end{array}\right) .
$$

Thus, boundary conditions at $r=r_{i}$ can be represented by

$$
M_{i}\left(r_{i}\right)\left(\begin{array}{c}
A^{(i)} \\
B^{(i)}
\end{array}\right)=M_{i+1}\left(r_{i}\right)\left(\begin{array}{c}
A^{(i+1)} \\
B^{(i+1)}
\end{array}\right), \quad 1<i<N-1 .
$$

Applying boundary conditions sequentially at boundaries $r=r_{n}, n=i+1, i+2, \ldots, N-1$, we can express arbitrary constants $A^{(i)}$ and $B^{(i)}$ through constants $A^{(N)}$ and $B^{(N)}$ thusly:

$$
\begin{aligned}
\left(\begin{array}{l}
A^{(i)} \\
B^{(i)}
\end{array}\right)= & M_{i}^{-1}\left(r_{i}\right) S^{(i+1)} S^{(i+2)} \cdots S^{(N-2)} M_{N-1}\left(r_{N-2}\right) \\
& \times\left(\begin{array}{l}
A^{(N-1)} \\
B^{(N-1)}
\end{array}\right)
\end{aligned}
$$

where the transition matrices $S^{(i)}\left(r_{i}\right)$ are defined as follows:

$$
S^{(i)}=M_{i}\left(r_{i-1}\right) M_{i}^{-1}\left(r_{i}\right)=\left(\begin{array}{ll}
S_{11}^{(i)}\left(r_{i-1}, r_{i}\right) & S_{12}^{(i)}\left(r_{i-1}, r_{i}\right) \\
S_{21}^{(i)}\left(r_{i-1}, r_{i}\right) & S_{22}^{(i)}\left(r_{i-1}, r_{i}\right)
\end{array}\right),
$$

with 


$$
\begin{aligned}
S_{11}^{(i)}\left(r_{i-1}, r_{i}\right)= & \frac{\pi}{2} \kappa_{i} r_{i}\left[J_{1}\left(\kappa_{i} r_{i-1}\right) Y_{0}\left(\kappa_{i} r_{i}\right)\right. \\
& \left.-Y_{1}\left(\kappa_{i} r_{i-1}\right) J_{0}\left(\kappa_{i} r_{i}\right)\right], \\
S_{12}^{(i)}\left(r_{i-1}, r_{i}\right)= & \frac{\pi}{2} \varepsilon_{i} r_{i}\left[J_{1}\left(\kappa_{i} r_{i-1}\right) Y_{1}\left(\kappa_{i} r_{i}\right)\right. \\
& \left.-Y_{1}\left(\kappa_{i} r_{i-1}\right) J_{1}\left(\kappa_{i} r_{i}\right)\right], \\
S_{21}^{(i)}\left(r_{i-1}, r_{i}\right)= & -\frac{\pi}{2} r_{i} \frac{\kappa_{i}^{2}}{\varepsilon_{i}}\left[J_{0}\left(\kappa_{i} r_{i-1}\right) Y_{0}\left(\kappa_{i} r_{i}\right)\right. \\
& \left.-Y_{0}\left(\kappa_{i} r_{i-1}\right) J_{0}\left(\kappa_{i} r_{i}\right)\right], \\
S_{22}^{(i)}\left(r_{i-1}, r_{i}\right)= & -\frac{\pi}{2} \kappa_{i} r_{i}\left[J_{0}\left(\kappa_{i} r_{i-1}\right) Y_{1}\left(\kappa_{i} r_{i}\right)\right. \\
& \left.-Y_{0}\left(\kappa_{i} r_{i-1}\right) J_{1}\left(\kappa_{i} r_{i}\right)\right] .
\end{aligned}
$$

In Eq. (7), the constants $A^{(N-1)}$ and $B^{(N-1)}$ are connected with the constants $A^{(N)}$ and $B^{(N)}$ in the outer layer of the dielectric structure by a relation similar to Eq. (6). But the constants $A^{(N)}$ and $B^{(N)}$ are not independent, and are connected by an additional condition, taking into account the outer border. Setting to zero the longitudinal electric field component at the outer conducting boundary surrounding the dielectric structure can be such a condition. The Sommerfeld radiation condition for the dielectric structure bounded by free space can be another possible condition. Not concretizing this condition before consideration of a specific dielectric structure, we will write down the boundary condition at $r=r_{N-1}$ as

$$
M_{N-1}\left(r_{N-1}\right)\left(\begin{array}{c}
A^{(N-1)} \\
B^{(N-1)}
\end{array}\right)=\left(\begin{array}{c}
1 \\
Z_{N}
\end{array}\right) A^{(N)},
$$

where the value $Z_{N}$ represents a surface impedance (to within a factor) and is given by

$$
Z_{N}=\frac{e_{z}^{(N)}\left(r_{N-1}\right)}{\frac{\varepsilon_{N}}{\kappa_{N}^{2}} \frac{\partial e_{z}^{(N)}\left(r_{N-1}\right)}{\partial r}} .
$$

Expressing constants $A^{(N-1)}$ and $B^{(N-1)}$ from Eq. (10) and substituting them into Eq. (7), we obtain

$$
\begin{aligned}
\left(\begin{array}{c}
A^{(i)} \\
B^{(i)}
\end{array}\right)= & M_{i}^{-1}\left(r_{i}\right) S^{(i+1)} S^{(i+2)} \cdots S^{(N-1)}\left(\begin{array}{c}
1 \\
Z_{N}
\end{array}\right) A^{(N)}, \\
& 1<i<N-1 .
\end{aligned}
$$

At the boundary of the 1st and 2nd zones we write down a condition from Eq. (10), namely,

$$
M_{2}\left(r_{1}\right)\left(\begin{array}{c}
A^{(2)} \\
B^{(2)}
\end{array}\right)=\left(\begin{array}{c}
1 \\
Z_{1}
\end{array}\right) A^{(1)}
$$

where

$$
Z_{1}=\frac{e_{z}^{(1)}\left(r_{1}\right)}{\frac{\varepsilon_{1}}{\kappa_{1}^{2}} \frac{\partial e_{z}^{(1)}\left(r_{1}\right)}{\partial r}} .
$$

Expressing constants $A^{(2)}$ and $B^{(2)}$ from Eq. (12) (with $i=2$ ), we substitute them into Eq. (13). After multiplication of the resulting matrix equation on the left by the row $\left(-Z_{1}, 1\right)$ we come to the final dispersion equation:

$$
\left(\begin{array}{ll}
-Z_{1}, & 1
\end{array}\right)\left(\prod_{n=2}^{N-1 \geq 2} S^{(n)}\right)\left(\begin{array}{c}
1 \\
Z_{N}
\end{array}\right)=0 .
$$

The dispersion equation (15) is valid for $N \geq 3$. But it can be generalized for a two-layer dielectric structure by interposing a layer with coincident radii (see Ref. [1]). Such an operation is similar to the condition that the product of transition matrices $S^{(i)}$ in Eq. (15) is equal to the unit matrix. The equation remains valid for a singlezone structure (when the external boundary is a conducting wall) if we furthermore set $Z_{2}=0$. In this manner, the equation is valid for any number of layers of a multizone dielectric structure.

We now provide examples for the expressions $Z_{1}$ and $Z_{N}$ for specific configurations of a multizone dielectric waveguide. If the dielectric waveguide has a sequence of concentric layers surrounded by a conducting pipe, then

$$
\begin{aligned}
Z_{N}= & \frac{\kappa_{N}}{\varepsilon_{N}} \\
& \cdot \frac{J_{0}\left(\kappa_{N} r_{N-1}\right) Y_{0}\left(\kappa_{N} r_{N}\right)-Y_{0}\left(\kappa_{N} r_{N-1}\right) J_{0}\left(\kappa_{N} r_{N}\right)}{-J_{1}\left(\kappa_{N} r_{N-1}\right) Y_{0}\left(\kappa_{N} r_{N}\right)+Y_{1}\left(\kappa_{N} r_{N-1}\right) J_{0}\left(\kappa_{N} r_{N}\right)} .
\end{aligned}
$$

If the dielectric structure is open to free space $\left(r_{N} \rightarrow \infty\right.$, $\varepsilon_{N}=1$ ), then the value $Z_{N}$ is described by

$$
Z_{N}=p_{N} \frac{K_{0}\left(p_{N} r_{N-1}\right)}{K_{1}\left(p_{N} r_{N-1}\right)},
$$

where $p_{N}=\sqrt{k_{z}^{2}-k^{2}}$, and $K_{0}$ and $K_{1}$ are the Macdonald functions of zero and first order.

If the first zone is a cylindrical $\left(r_{0}=0\right)$ vacuum channel then the impedance $Z_{1}$ at its outer surface $r=r_{1}$ is given by

$$
Z_{1}=-p_{1} \frac{I_{0}\left(p_{1} r_{1}\right)}{I_{1}\left(p_{1} r_{1}\right)}
$$

where $p_{1}=\sqrt{k_{z}^{2}-k^{2}}$, and $I_{0}$ and $I_{1}$ are the modified Bessel functions of a zero and first order.

Solutions of the dispersion equation (15) for fixed longitudinal wave number $k_{z}$ give eigenfrequencies $\omega=$ $\omega_{m}\left(k_{z}\right)$, and at fixed frequency $\omega$ give eigen-wavenumbers $k_{z}=k_{m}(\omega)$. However, in the case of the excitation of a dielectric waveguide by a moving charge, the frequencies and wave numbers are connected by an equiphase condition $\omega_{m}=k_{m} v_{0}$ (where $v_{0}$ is the velocity of the charge). Subject to this proviso the dispersion equation (15) gives frequencies and wave numbers for eigenmodes that comprise Cherenkov (wakefield) radiation in 
dielectric structures that must move synchronously with the drive charges. Having found from Eq. (4) with the use of Eq. (12) the quantities $e_{z}^{(i)}$ and $e_{r}^{(i)}$, and having replaced in the derived expressions the frequency $\omega$ and the wave vector $k_{z}$ by their eigenvalues, we obtain the following expressions for the electric field intensities of the eigenwaves inside the $i$ th layer $(1<i<N)$ :

$$
\begin{gathered}
e_{z}^{(i)}=\left[S_{21}^{(i)}\left(r, r_{i}\right), S_{22}^{(i)}\left(r, r_{i}\right)\right] \Lambda_{m}^{(i)}, \\
e_{r}^{(i)}(r)=\frac{k_{m}}{\varepsilon^{(i)}}\left[S_{11}^{(i)}\left(r, r_{i}\right), S_{12}^{(i)}\left(r, r_{i}\right)\right] \Lambda_{m}^{(i)} .
\end{gathered}
$$

For determination of fields in the first zone we shall use Eq. (13). After simple transformations we find

$$
\begin{aligned}
& e_{z}^{(1)}(r)=\varphi_{z}^{(1)}(r) \cdot(1,0) \Lambda_{m}^{(1)}, \\
& e_{r}^{(1)}(r)=\varphi_{r}^{(1)}(r) \cdot(1,0) \Lambda_{m}^{(1)} .
\end{aligned}
$$

In expressions (19)-(21) the quantity $\Lambda_{m}^{(i)}$ is defined as follows:

$$
\Lambda_{m}^{(i)}=A^{(N)} \begin{cases}\left(\prod_{n=i+1}^{N-1 \geq 2} S^{(n)}\right)\left(\begin{array}{c}
1 \\
Z_{N}
\end{array}\right), & 1 \leq i<N-1 \\
\left(\begin{array}{c}
1 \\
Z_{N}
\end{array}\right), & i=N-1 .\end{cases}
$$

Functions $\varphi_{z}^{(1)}(r)$ and $\varphi_{r}^{(1)}(r)$ which define the radial dependence of fields in the first zone are normalized so as to satisfy Eq. (13), as follows:

$$
\varphi_{z}^{(1)}\left(r=r_{1}\right)=Z_{1}, \quad \varphi_{r}^{(1)}\left(r=r_{1}\right)=1 .
$$

Considering boundary conditions and Eq. (10), we write down fields in the remaining $N$ th layer of the structure in the form

$$
e_{z}^{(N)}(r)=\varphi_{z}^{(N)}(r) A^{(N)}, \quad e_{r}^{(N)}(r)=\varphi_{r}^{(N)}(r) A^{(N)},
$$

where the functions $\varphi_{z}^{(N)}(r)$ and, $\varphi_{r}^{(N)}(r)$ satisfy the conditions

$$
\varphi_{Z}^{(N)}\left(r=r_{N-1}\right)=Z_{N}, \quad \varphi_{r}^{(N)}\left(r=r_{N-1}\right)=1 .
$$

We present as examples the expressions for functions $\varphi_{r, z}^{(1, N)}$ of some typical configurations. If the first zone is a vacuum cylindrical channel, then

$$
\varphi_{z}^{(1)}=-p_{1} \frac{I_{0}\left(p_{1} r\right)}{I_{1}\left(p_{1} r_{1}\right)}, \quad \varphi_{r}^{(1)}=\frac{I_{1}\left(p_{1} r\right)}{I_{1}\left(p_{1} r_{1}\right)} .
$$

If the cylindrical multizone dielectric structure is surrounded by a metal sheath, then the radial structure of fields in the last zone with index $N$ is described by expressions

$$
\begin{aligned}
\varphi_{z}^{(N)}= & \frac{\kappa_{N}}{\varepsilon_{N}} \\
& \cdot \frac{J_{0}\left(\kappa_{N} r\right) Y_{0}\left(\kappa_{N} r_{N}\right)-Y_{0}\left(\kappa_{N} r\right) J_{0}\left(\kappa_{N} r_{N}\right)}{-J_{1}\left(\kappa_{N} r_{N-1}\right) Y_{0}\left(\kappa_{N} r_{N}\right)+Y_{1}\left(\kappa_{N} r_{N-1}\right) J_{0}\left(\kappa_{N} r_{N}\right)},
\end{aligned}
$$

$$
\varphi_{r}^{(N)}=\frac{J_{1}\left(\kappa_{N} r\right) Y_{0}\left(\kappa_{N} r_{N}\right)-Y_{1}\left(\kappa_{N} r\right) J_{0}\left(\kappa_{N} r_{N}\right)}{J_{1}\left(\kappa_{N} r_{N-1}\right) Y_{0}\left(\kappa_{N} r_{N}\right)-Y_{1}\left(\kappa_{N} r_{N-1}\right) J_{0}\left(\kappa_{N} r_{N}\right)} .
$$

Finally, if the dielectric structure is surrounded by free space,

$$
\varphi_{z}^{(N)}=p_{N} \frac{K_{0}\left(p_{N} r\right)}{K_{1}\left(p_{N} r_{N-1}\right)}, \quad \varphi_{r}^{(N)}=\frac{K_{1}\left(p_{N} r\right)}{K_{1}\left(p_{N} r_{N-1}\right)} .
$$

\section{WAKEFIELD OF A THIN ANNULAR BUNCH}

Next we find the fields (Green's function) that are excited in a multizone coaxial dielectric structure by a charged particle bunch having the shape of an infinitesimally thin ring. The density of particles of such a bunch is described by the expression

$$
\rho=\frac{Q}{2 \pi r} \delta\left(r-r_{b}\right) \delta\left(z-z_{0}-v_{0} t\right),
$$

where $r_{b}$ is the radius of the annular bunch, $z_{0}$ is its position at the moment of time $t=0, \delta(x)$ is the Dirac delta function, $v_{0}$ is the bunch velocity along $z$, and $Q$ is charge of a bunch.

Neglecting entry and exit boundary effects, we can consider that the excited electromagnetic fields are defined only by this external source. Therefore, the dependence of fields upon time and longitudinal coordinates is defined only by the combined variable $\xi=v_{0} t-z$. Then, from Maxwell equations we obtain the following inhomogeneous differential equation for determining the longitudinal electric field:

$$
\frac{1}{r} \frac{\partial}{\partial r}\left[\frac{\varepsilon r}{1-\beta_{0}^{2} \varepsilon \mu} \frac{\partial E_{z}^{G}}{\partial r}\right]+\varepsilon \frac{\partial^{2} E_{z}^{G}}{\partial \xi^{2}}=-4 \pi \frac{\partial \rho}{\partial \xi} .
$$

Here $\beta_{0}=v_{0} / c$ and the dielectric permittivity depends on radius, $\varepsilon=\varepsilon(r), \mu=\mu(r)$, i.e., the equation is true for any axisymmetric radially nonuniform dielectric structure. In the case we study here, $\varepsilon=\varepsilon_{i}, \mu=1$ when $r_{i-1} \leq$ $r<r_{i}$.

A Fourier transformation with respect to the variable $\xi$ is then carried out, namely,

$$
\left(E_{z}^{k}, \rho^{k}\right)=\frac{1}{2 \pi} \int_{-\infty}^{\infty} d \xi\left(E_{z}^{G} \rho\right) \exp \left[i k_{z} \xi\right]
$$

from which we obtain the equation for finding the Fourier amplitudes of the longitudinal electric field: 


$$
\frac{1}{r} \frac{\partial}{\partial r}\left[\frac{\varepsilon r}{1-\beta_{0}^{2} \varepsilon \mu} \frac{\partial E_{z}^{k}}{\partial r}\right]-\varepsilon k_{z}^{2} E_{z}^{k}=4 \pi i k_{z} \rho^{k},
$$

where

$$
\rho^{k}=\frac{Q}{4 \pi^{2} r} \delta\left(r-r_{b}\right) \exp \left[-i k_{z} z_{0}\right]
$$

We will find the solution of Eq. (33) by decomposition into waves, i.e.,

$$
E_{z}^{k}=\sum_{m} a_{m} e_{z m}(r) .
$$

Having substituted Eq. (35) into the left side of Eq. (33) and having multiplied both sides of the equation by the complex conjugate eigenfunction $e_{z n}^{*}(r)$, and having integrated both sides of the equation over the cross section of a multilayered dielectric structure, we obtain for the decomposition coefficients $a_{m}$ the expression

$$
a_{m}=-\frac{i k_{z}}{k_{z}^{2}-k_{m}^{2}} \frac{Q}{\pi r_{N-1}^{2}} \frac{e_{z m}^{*}\left(r_{b}\right)}{\left\|e_{z m}\right\|^{2}} \exp \left[-i k_{z} z_{0}\right] .
$$

In deriving Eq. (36) we made use of the orthogonality condition

$$
\frac{1}{r_{N-1}^{2}} \int_{r_{0}}^{r_{N}} d r \cdot r \varepsilon(r) e_{z m}(r) e_{z n}^{*}(r)=\left\|e_{z m}\right\|^{2} \delta_{m n},
$$

and that the eigenfunction $e_{z m}(r)$ satisfies the equation:

$$
\frac{1}{r} \frac{\partial}{\partial r}\left[\frac{\varepsilon r}{1-\beta_{0}^{2} \varepsilon \mu} \frac{\partial e_{z m}(r)}{\partial r}\right]-\varepsilon k_{m}^{2} e_{z m}(r)=0,
$$

where $\delta_{m n}$ is the Kronecker symbol, and $\left\|e_{z m}\right\|^{2}$ is the normalizing factor of the eigenfunction $e_{z m}(r)$.

The orthogonality relation Eq. (37) is similar to that found in [2], and applies for eigenwaves with equal phase velocities (appropriate for Cherenkov excitation) in a multilayered dielectric structure. But, it was derived in [2] a cumbersome way starting with the overlap integral for transverse components of electric and magnetic fields in a particular dielectric layer. By way of transformation of this integral and summation of overlap integrals of all layers, it was reduced to the orthogonality relation for axial components of electric and a magnetic fields. The general notation of the relation Eq. (38), valid in any layer, with use of nonuniform dielectric permittivity and magnetic permeability allows one to obtain at once, and in a simpler way, the orthogonality relation for the axial component of electric and magnetic fields. Thus, we write the equation similar to Eq. (38) for a complex conjugate function $e_{z n}^{*}(r)$, multiply it by $e_{z m}(r)$, and subtract it from the equation (38) multiplied by $e_{z n}^{*}(r)$. After integration of the obtained expression by parts we arrive at an orthogonality condition. It is necessary to make use of the following boundary conditions: for the last zone $e_{z m}\left(r=r_{N}\right)=0$; for the first zone $e_{z m}\left(r=r_{0}\right)=0$, if $r_{0} \neq 0$, or $e_{r m}\left(r=r_{0}\right)=0$, if $r_{0}=0$.
Substituting Eq. (36) into Eq. (35) and executing the inverse Fourier transform, we obtain the final expression for the longitudinal component of the electric field for the annular bunch:

$$
E_{z}^{G}(r, \xi)=-\frac{2 Q}{r_{N-1}^{2}} \sum_{m} \frac{e_{z m}(r) e_{z m}^{*}\left(r_{b}\right)}{\left\|e_{z m}\right\|^{2}} \Psi_{z m}\left(\xi+z_{0}\right),
$$

where

$$
\begin{gathered}
\Psi_{z m}(\xi)= \begin{cases}\cos \left(k_{m} \xi\right) \theta(\xi), & k_{m}^{2}>0 \\
X_{\| m}(\xi), & k_{m}^{2}<0\end{cases} \\
X_{\| m}(\xi)=\frac{1}{2}\left[e^{-\left|k_{m}\right| \xi} \theta(\xi)-e^{\left|k_{m}\right| \xi} \theta(-\xi)\right],
\end{gathered}
$$

$\theta(\xi)$ is the Heaviside step function. The function $\mathrm{X}_{\| m}(\xi)$ describes the axial profile of longitudinal (nonradiating) electric field in the case of purely imaginary solutions of the dispersion equation that corresponds to the (self) Coulomb field of a charge, present with or without Cherenkov radiation.

After deriving the expression for the longitudinal field $E_{z}^{G}(r, \xi)$, the other components of the electromagnetic field are obtained from the Maxwell equations, as follows:

$$
E_{r}^{G}(r, \xi)=\frac{2 Q}{r_{N-1}^{2}} \sum_{m} \frac{e_{r m}(r) e_{z m}^{*}\left(r_{b}\right)}{\left\|e_{z m}\right\|^{2}} \Psi_{\perp m}\left(\xi+z_{0}\right),
$$

$$
H_{\varphi}^{G}(r, \xi)=\beta_{0} E_{r}^{G}(r, \xi)
$$

where

$$
\begin{array}{r}
\Psi_{\perp m}(\xi)= \begin{cases}\sin \left(k_{m} \xi\right) \theta(\xi), & k_{m}^{2}>0 \\
X_{\perp m}(\xi), & k_{m}^{2}<0\end{cases} \\
X_{\perp m}(\xi)=\frac{1}{2 i}\left[e^{-\left|k_{m}\right| \xi} \theta(\xi)+e^{\left|k_{m}\right| \xi} \theta(-\xi)\right] .
\end{array}
$$

The wakefield energized by finite-size annular bunches is obtained by summation of the fields from infinitesimally thin ring bunches. Let the charge distribution in a bunch be described by the separable function $\rho_{b}\left(r_{b}, z_{0}\right)=$ $\rho_{\|}\left(z_{0}\right) \rho_{\perp}\left(r_{b}\right)$. Then,

$$
\left(E_{r}, E_{z}, H_{\varphi}\right)=\iint_{V_{b}} d z_{0} d r_{b} r_{b}\left(E_{r}^{G}, E_{z}^{G}, H_{\varphi}^{G}\right) \rho_{b}\left(r_{b}, z_{0}\right),
$$

with

$$
\int d z_{0} \rho_{\|}\left(z_{0}\right)=1, \quad \int d r_{b} r_{b} \rho_{\perp}\left(r_{b}\right)=1 .
$$

Thus, for a finite-size bunch we can write down the field components in the following form:

$$
E_{z}(r, \xi)=-\frac{2 Q}{r_{N-1}^{2}} \sum_{m} \frac{R_{m}}{\left\|e_{z m}\right\|^{2}} \cdot e_{z m}(r) Z_{\| m}(\xi),
$$


$E_{r}(r, \xi)=\frac{2 Q}{r_{N-1}^{2}} \sum_{m} \frac{R_{m}}{\left\|e_{z m}\right\|^{2}} \cdot e_{r m}(r) Z_{\perp m}(\xi)$,

$H_{\varphi}(r, \xi)=\beta_{0} E_{r}(r, \xi)$,

where

$$
\begin{gathered}
Z_{\|, \perp m}(\xi)=\int d z_{0} \rho_{\|}\left(z_{0}\right) \Psi_{\|, \perp m}\left(\xi+z_{0}\right) \\
R_{m}=\int d r_{b} \cdot r_{b} \rho_{\perp}\left(r_{b}\right) e_{z m}^{*}\left(r_{b}\right) .
\end{gathered}
$$

For excitation of the dielectric structure by a sequence of $N_{b}$ collinear bunches with a Gaussian distribution of charge in each bunch, one has

$$
\begin{gathered}
\rho_{\|}\left(z_{0}\right)=\frac{1}{\sqrt{2 \pi} \sigma_{z}} \sum_{n=1}^{N_{b}} \exp \left[-\frac{\left[z_{0}+(n-1) L_{m}\right]^{2}}{2 \sigma_{z}^{2}}\right], \\
\rho_{\perp}\left(r_{b}\right)=\frac{1}{\sigma_{r}^{2}} \exp \left[-\frac{\left(r_{b}-r_{c}\right)^{2}}{2 \sigma_{r}^{2}}\right]
\end{gathered}
$$

for functions $Z_{\|, \perp m}$ and $R_{m}$ we find

$$
Z_{\| m}(\xi)=\sum_{n=1}^{N_{b}} C_{m} \cos \left(k_{m} \xi_{n}\right)+S_{m} \sin \left(k_{m} \xi_{n}\right), \quad k_{m}^{2}>0
$$

$$
\begin{gathered}
Z_{\| m}(\xi)=\sum_{n=1}^{N_{b}} C_{m}^{h} e^{-\left|k_{m}\right| \xi_{n}}-S_{m}^{h} e^{\left|k_{m}\right| \xi_{n}}, \quad k_{m}^{2}<0 \\
Z_{\perp m}(\xi)=\sum_{n=1}^{N_{b}} C_{m} \sin \left(k_{m} \xi_{n}\right)-S_{m} \cos \left(k_{m} \xi_{n}\right), \quad k_{m}^{2}>0
\end{gathered}
$$

$$
Z_{\perp m}(\xi)=-i \sum_{n=1}^{N_{b}} C_{m}^{h} e^{-\left|k_{m}\right| \xi_{n}}+S_{m}^{h} e^{\left|k_{m}\right| \xi_{n}}, \quad k_{m}^{2}<0
$$

$$
R_{m}=\frac{1}{\sigma_{r}^{2}} \int_{r_{l-1}}^{r_{l}} d r_{b} r_{b} \cdot e_{z m}^{*}\left(r_{b}\right) \exp \left[-\frac{\left(r_{b}-r_{c}\right)^{2}}{2 \sigma_{r}^{2}}\right],
$$

where

$$
\begin{array}{r}
C_{m}=\frac{1}{\sqrt{2 \pi}} \int_{-\infty}^{\xi_{n} / \sigma_{z}} d x \cos \left(k_{m} \sigma_{z} x\right) e^{-x^{2} / 2}, \\
S_{m}=\frac{1}{\sqrt{2 \pi}} \int_{-\infty}^{\xi_{n} / \sigma_{z}} d x \sin \left(k_{m} \sigma_{z} x\right) e^{-x^{2} / 2}, \\
C_{m}^{h}=\frac{1}{2 \sqrt{2 \pi}} \int_{-\infty}^{\xi_{n} / \sigma_{z}} d x \exp \left[\left|k_{m}\right| \sigma_{z} x-x^{2} / 2\right],
\end{array}
$$

$$
S_{m}^{h}=\frac{1}{2 \sqrt{2 \pi}} \int_{\xi_{n} / \sigma_{z}}^{\infty} d x \exp \left[-\left|k_{m}\right| \sigma_{z} x-x^{2} / 2\right],
$$

$\xi_{n}=\xi-(n-1) L_{m}, L_{m}$ is the space bunch-repetition interval, $\sigma_{z}$ and $\sigma_{r}$ are the longitudinal and transverse rms bunch dimensions, $r_{c}$ is the bunch center radius, and $l$ is the zone number in which these drive bunches travel.

For the numerical study which is described below, we shall use bunches having a rectangular transverse density profile $\rho_{\perp}\left(r_{b}\right)$. Such a profile is often used in a full numerical simulation by a particle-in-cell (PIC) code. In this case the quantity $R_{m}$ should be calculated according to the expression

$$
R_{m}=\frac{2}{\left(r_{b 2}^{2}-r_{b 1}^{2}\right) k_{m}^{*}}\left[r_{b 2} e_{r m}^{*}\left(r_{b 2}\right)-r_{b 1} e_{r m}^{*}\left(r_{b 1}\right)\right],
$$

where $r_{b 2}$ and $r_{b 1}$ are the outer and inner bunch radii.

Radiation power loss for a finite-size bunch can be obtained by integration of the expression for radiation losses of each particle of the bunch integrated over the volume occupied by the bunch, i.e. $P_{\text {rad }}=$ $-\int_{V_{b}} \rho v_{0} E_{z} d V . P_{\text {rad }}$ is equal to the work of retarding forces acting on particles from the fields excited by the bunch itself:

$$
P_{\mathrm{rad}}=\sum_{m, k_{m}^{2}>0} P_{m}=\frac{2 Q^{2} v_{0}}{r_{N-1}^{2}} \sum_{m, k_{m}^{2}>0} \frac{\left|R_{m}\right|^{2}}{\left\|e_{z m}\right\|^{2}} \cdot \Phi_{m},
$$

where

$$
\Phi_{m}=\int_{-L_{b} / 2}^{L_{b} / 2} d \xi \rho_{\|}(\xi) Z_{\| m}(\xi)
$$

In the case of the longitudinal distribution of density of a bunch according to Gauss's law in Eq. (63), the limits of integration $-L_{b} / 2$ and $L_{b} / 2$ should be replaced by $-\infty$ and $\infty$.

Equation (63) for energy losses allows one to define the average braking force or drag acting on an elementary charge $q$ within a bunch:

$$
F_{\mathrm{drag}}=\frac{2 q Q}{r_{N-1}^{2}} \sum_{m, k_{m}^{2}>0} \frac{\left|R_{m}\right|^{2}}{\left\|e_{z m}\right\|^{2}} \cdot \Phi_{m}
$$

Using the definition for the transformer ratio $T$ given in the Introduction, we find, from Eqs. (48) and (65),

$$
T\left(r=r_{\mathrm{ac}}, \xi\right)=-\frac{\sum_{m} R_{m} Z_{\| m}(\xi) e_{z m}\left(r=r_{\mathrm{ac}}\right) /\left\|e_{z m}\right\|^{2}}{\sum_{m, k_{m}^{2}>0}\left|R_{m}\right|^{2} \Phi_{m} /\left\|e_{z m}\right\|^{2}} .
$$

In Eq. (66), the sign on an elementary charge of an accelerated test particle is taken to coincide with the sign of the charges of the drive bunch, and $r_{\text {ac }}$ is the radial position of a test particle. 


\section{NUMERICAL RESULTS}

Using the analytical expressions for wakefields derived in the previous sections, numerical calculations relevant to specific coaxial dielectric accelerating structures have been carried out. These are described and discussed here. The simplest multizone coaxial structure is a four-zone structure having vacuum channels for the drive and witness bunches and two dielectric shells. A schematic of this structure is shown in Fig. 1.

To have much practical relevance, an accelerating module should show promise for efficient high-energy gain of accelerated particles, so that many (but not too many) such modules can be arranged to achieve electron or positron acceleration into the $\mathrm{TeV}$ energy range. We take for numerical calculations an annular drive bunch with charge $(6 \mathrm{nC})$ and energy $(5 \mathrm{GeV})$ similar to the SLAC bunch [12]. We ask what test bunch energy could be achieved by using a sequence of 1-m long DWFA modules, each with an effective acceleration gradient $G=500 \mathrm{MeV} / \mathrm{m}$ and a transformer ratio $T \sim 10$. Taking a drive bunch energy loss of $50 \mathrm{MeV}$ per module would allow traversal of nearly 100 modules by the drive bunch before energy exhaustion, with an overall energy gain of about $50 \mathrm{GeV}$ by the test bunch. After 10 similar groups of structures each fed by $5 \mathrm{GeV}$ drive bunches (1000 modules altogether) in a total length $\sim 1 \mathrm{~km}$, the test beam energy would ideally be $0.5 \mathrm{TeV}$. The challenge is to devise a module with $G=$ $500 \mathrm{MeV} / \mathrm{m}$ and $T \sim 10$. As shall be shown, this challenge could be met in the single-bunch mode using a mm-scale coaxial DWFA structure.

To obtain such high accelerating gradients it is necessary to use ultrahigh-frequency structures having a small cross section. At a given frequency of a wakefield mode, the necessary cross-section dimensions of the dielectric layers are determined from the dispersion equation (15), together with Eqs. (16) and (18). It is necessary to observe that the frequency and wave vector are connected by the Cherenkov resonance condition $\omega_{m}=k_{m} v_{0}$. In Table I the dimensions of the dielectric shells calculated for an operating frequency of $912 \mathrm{GHz}$ are listed. The symmetric $E_{02}$ mode is chosen, for which the longitudinal field inten- sity has a different sign in the drive bunch channel and the accelerating channel. We use the following simplifying method to optimize the dimensions of the dielectric layers, similar to that used to find the necessary cross-section sizes of a rectangular two-channel [15] and a symmetric threechannel [16] dielectric structure. Suppose that, at the radius of the vanishing of longitudinal electric field in a dielectric shell, we place a metal cylindrical surface; this will not change the structure of the $E_{02}$-mode fields. Thus, we can split our four-zone structure into a two-zone one (an interior dielectric waveguide with its internal vacuum channel) and a three-zone one (a coaxial metal waveguide with dielectric layers on the internal and external conductors). Having found the necessary zone dimensions, we can perform the reverse procedure, i.e., assemble the required four-zone structure from these two-zone and three-zone structures. In order to obtain a high transformer ratio, it is necessary that the radial thickness of the drive bunch channel be considerably larger than the radius of the accelerating channel.

The thicknesses of the dielectric shells, and the crosssection sizes of the vacuum channels are shown in Table I; these coincide with the thickness of dielectric plates and width of vacuum channels in a symmetric terahertz dielectric rectangular accelerating structure, recently investigated by us [16]. The latter provided a working frequency of $1.003 \mathrm{THz}$. Two synchronously moving bunches with energy of $5 \mathrm{GeV}$ and a charge of $3 \mathrm{nC}$ each developed accelerating gradients $G \sim 350 \mathrm{MeV} / \mathrm{m}$ in that structure. For the computation results described below for the coaxial device, the same drive bunch parameters are chosen except that a single annular drive bunch having total charge of $6 \mathrm{nC}$ is used.

In Fig. 3, radial profiles of the longitudinal electric field amplitudes of the dominant $E_{0 m}$ modes of the wakefield are shown. The radial dependence of $E_{z}$ is uniform inside the vacuum spaces and steps up in the dielectric layers. The ratio of the field amplitude in the accelerating channel to the field amplitude in the drive bunch channel for the $E_{02}$ mode is equal to 32.5. For phase synchronism with a $5 \mathrm{GeV}$ drive bunch, the frequencies of the first six modes in

TABLE I. Parameters for a two-channel coaxial dielectric wakefield accelerator module.

\begin{tabular}{lc}
\hline \hline Frequency of the $E_{02}$ design mode & $912.459 \mathrm{GHz}$ \\
External radius of outer coaxial dielectric shell $r_{4}$ & $1060.5 \mu \mathrm{m}$ \\
Inner radius of outer coaxial dielectric shell $r_{3}$ & $1047.5 \mu \mathrm{m}$ \\
External radius of inner coaxial dielectric shell $r_{2}$ & $89.5 \mu \mathrm{m}$ \\
Accelerating channel radius (inner radius of inner coaxial dielectric shell) $r_{1}$ & $50.0 \mu \mathrm{m}$ \\
Relative dielectric constant of dielectric shells $\varepsilon$ & 5.7 \\
rms bunch length $\sigma_{z}$ (Gaussian charge distribution) & $34.64 \mu \mathrm{m}$ \\
Outer drive bunch radius (box charge distribution) $r_{b 2}$ & $718.5 \mu \mathrm{m}$ \\
Inner drive bunch radius $r_{b 1}$ & $418.5 \mu \mathrm{m}$ \\
Bunch energy & $5 \mathrm{GeV}$ \\
Bunch charge & $6 \mathrm{nC}$ \\
\hline \hline
\end{tabular}




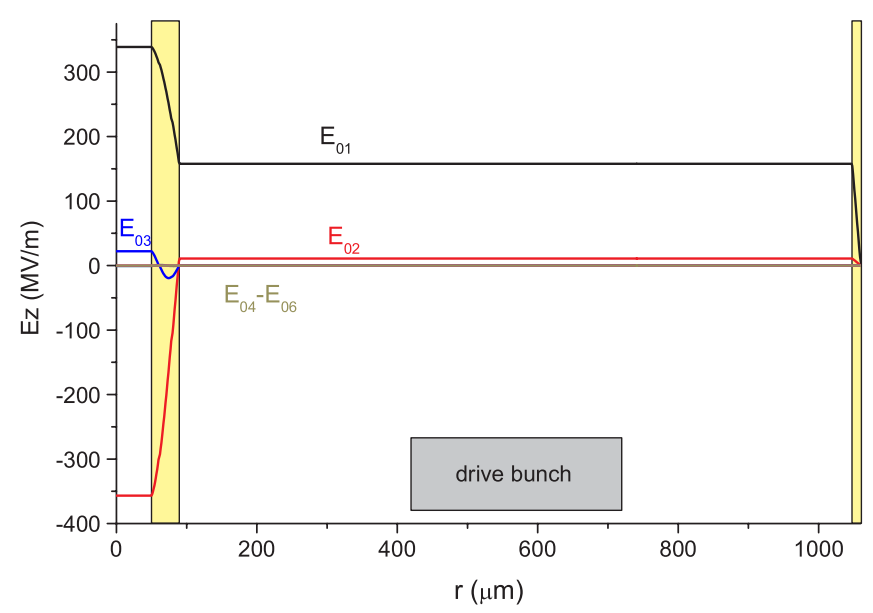

FIG. 3. (Color) Transverse profiles $E_{z}(r)$ for the first six TM modes, with the $E_{02}$ operating mode. Locations of the two dielectric shells is highlighted in yellow, and location and width of the $5-\mathrm{GeV}$ drive bunch is shown in gray.

ascending order are $611.252 \mathrm{GHz}\left(E_{01}\right), 912.459 \mathrm{GHz}$ $\left(E_{02}\right), 2417.845 \mathrm{GHz}\left(E_{03}\right), 4018.595 \mathrm{GHz}\left(E_{04}\right)$, $5393.728 \mathrm{GHz}\left(E_{05}\right)$, and $5665.204 \mathrm{GHz}\left(E_{06}\right)$. Only the first three of these modes have appreciable field strength in the accelerating channel; the amplitudes of the $E_{01}$ and the $E_{02}$ modes at the structure axis are $\sim 350 \mathrm{MV} / \mathrm{m}$.

In Fig. 4 the axial profiles of the composite longitudinal force along the axis of the accelerating channel are shown. The maximum [17] of the accelerating gradient $G$ of $587.6 \mathrm{MeV} / \mathrm{m}$ is located at about $306 \mu \mathrm{m}$ behind the drive bunch center. The transformer ratio at this maximum, according to Eq. (66), is 7.74. Although this value of transformer ratio is high, it is significantly less than the transformer ratio calculated using only the $E_{02}$ mode (see Fig. 3). The reason is the strong coupling of the $E_{01}$ and the $E_{02}$ modes, which are approximately equal in absolute

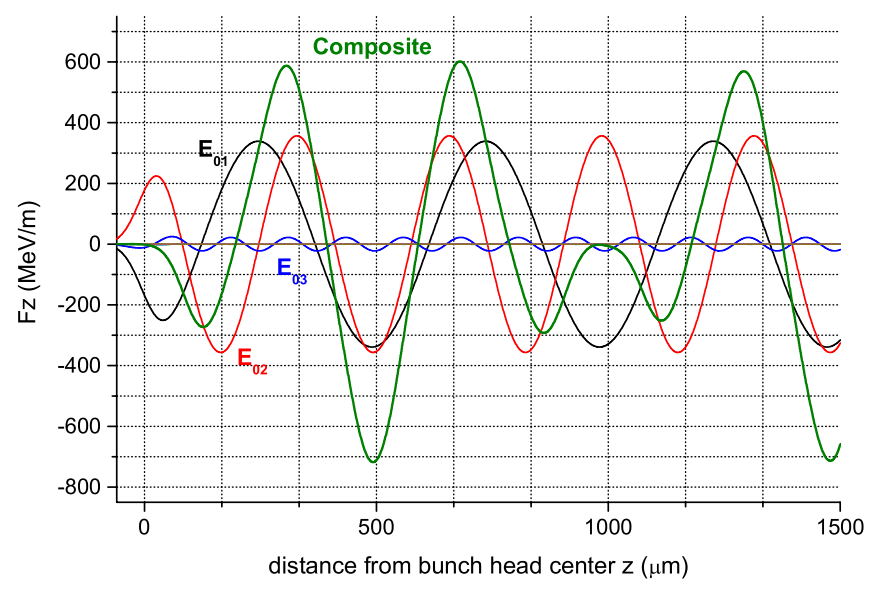

FIG. 4. (Color) Axial profiles of the longitudinal composite force and components of the force of the $E_{0 m}$ modes acting on a test electron along center of accelerating channel. The drive bunch moves from right to left and its center is located at $z=0$.

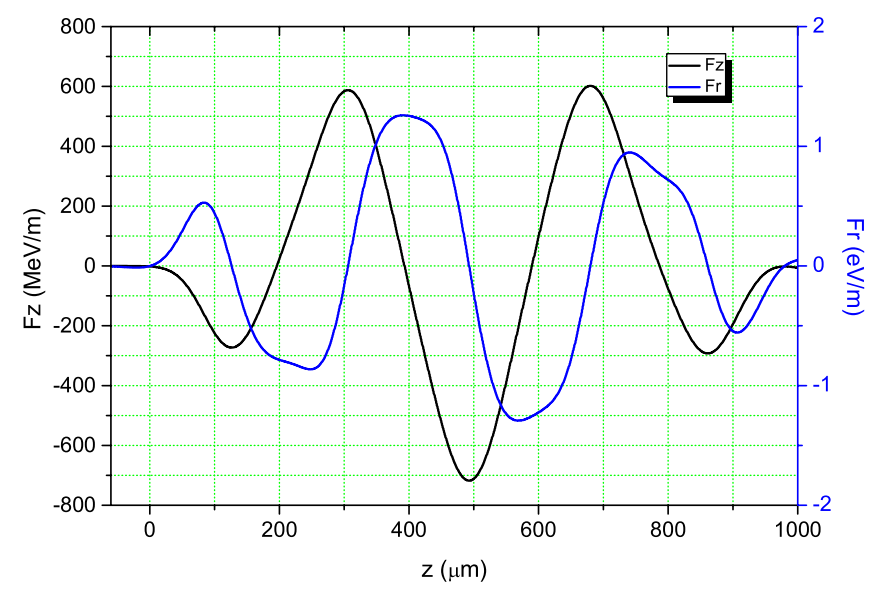

FIG. 5. (Color) Axial profiles of the composite transverse force (blue line) and composite longitudinal force (black line) acting on a test electron at the distance of $25 \mu \mathrm{m}$ from center of the accelerating channel. The drive bunch moves from right to left and its center is located at $z=0$. Parameters of the structure and drive bunch are given in the Table I.

value but opposite in sign in the witness channel. By carrying out computations using different dimensions of the coaxial dielectric-loaded structures (CDS), we find that it is impossible to suppress the $E_{01}$ mode appreciably and, thereby increase the transformer ratio.

The axial profile of the transverse force acting on a test electron, moving at a distance $25 \mu \mathrm{m}$ from the axis of the accelerating channel, is shown in Fig. 5. The transverse force at the maximum of the accelerating field is equal to zero. If the center of a test bunch is placed at the maximum of this accelerating field, the transverse force will be focusing for the bunch head and defocusing for the bunch tail and that will lead to slight pinching of the bunch. As follows from Fig. 5, it is desirable to place a witness bunch a little ahead of the maximum of the accelerating field. Although there is some loss in acceleration, the radial force there is focusing and thus there is better transverse stability of the witness bunch. But even if we place a witness bunch at exactly the maximum of the accelerating field, the radial forces there are not dangerous for accelerating modules having a small length, since they are insignificant in absolute value. For example, if we take the accelerating module to have a length $1 \mathrm{~m}$ (see above), a test electron, located $34.6 \mu \mathrm{m}$ behind the maximum of the accelerating field (same rms length as the drive bunch) and at distance $25 \mu \mathrm{m}$ from an axis, will deflect only $0.85 \mu \mathrm{m}$ in the transverse direction.

The simplified procedure to choose the dimensions of coaxial dielectric structure described above is preliminary as it uses only one operating mode of the excited field (in our example, the $E_{02}$ ). However, the reference structure is excited not only by the operating mode which is an eigenmode for the partial structures into which we have split the reference structure, but also other modes. The composite 


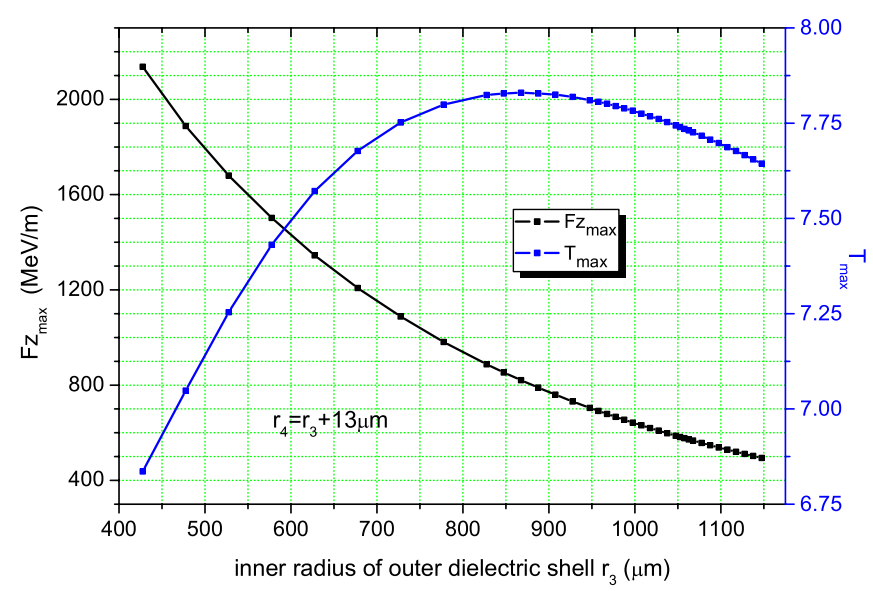

FIG. 6. (Color) The value of the composite longitudinal electric field at the first axial maximum and corresponding transformer ratio versus the dimension of the drive channel. The thickness of the outer dielectric shell is fixed and equal $13 \mu \mathrm{m}$. The other parameters are the same as those in Table I.

field is a complex superposition of all excited modes and, therefore, to obtain the best characteristics of this accelerating structure it is necessary to optimize its parameters. Such optimization and finding characteristics of the structure depends on the dimensions of vacuum channels and dielectric layers, as discussed below.

The dependence of the accelerating gradient on the width of the drive channel is presented in Fig. 6. With decreasing drive channel width the maximum of the accelerating gradient grows approximately in inverse proportion to the square of the external radius of the channel. The transformer ratio changes little for considerable change of width of the channel. The transformer ratio varies gradually from 6.83 to 7.83 as the width of the vacuum channel changes from 338 to $1048 \mu \mathrm{m}$. The coaxial wakefield accelerating structure differs from the rectangular accelerating structure $[15,16]$ where the transformer ratio strongly depends on width of drive channel. We also note that the transverse forces operating at the accelerated test bunch increase with decreasing drive channel width approximately in the same proportion as the accelerating field.

While decreasing the drive channel width, the longitudinal distance to the first maximum from the center of a drive bunch shortens (see Fig. 7). This shortening is not large and, as follows from Fig. 8, it is connected with the decrease of wavelength of the first two radial harmonics of the field.

From Figs. 6-8, one can propose an accelerating structure for which the inner and external radii of the outer dielectric shell are $r_{3}=777.5 \mu \mathrm{m}$ and $r_{4}=790.5 \mu \mathrm{m}$, and the inner and outer radii of the inner dielectric shell are $r_{1}=50 \mu \mathrm{m}$ and $r_{2}=89.5 \mu \mathrm{m}$. With these dimensions it is possible to obtain an accelerating field $\sim 1 \mathrm{GeV} / \mathrm{m}$ at a distance of $293 \mu \mathrm{m}$ behind the center of the drive bunch. However, for technical reasons it might be desirable to use

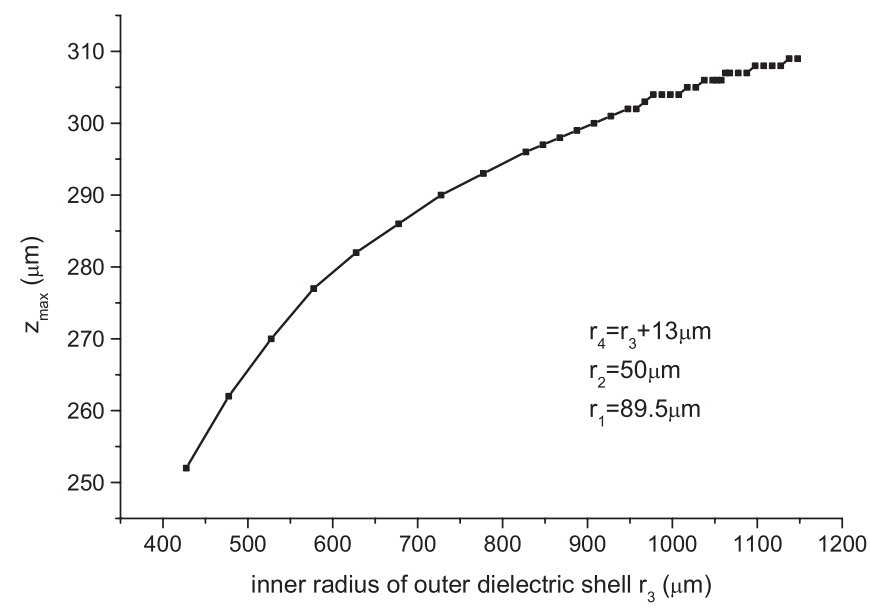

FIG. 7. The location (from drive bunch center) of the first maximum of the accelerating field versus the width of the drive channel. The thickness of the outer dielectric shell is fixed and equal to $13 \mu \mathrm{m}$. The other parameters are as in Table I.

thicker dielectric tubes as they are more sturdy and are simpler to manufacture and mount.

In Fig. 9, we show the dependence of the accelerating field and transformer ratio at the first axial maximum upon the external radius of the inner dielectric tube $r_{2}$. Upon increasing, the increase in $r_{2}$ from its original size to $110 \mu \mathrm{m}$, there is an abrupt decrease of the accelerating gradient to $775 \mathrm{MeV} / \mathrm{m}$. A further increase in the thickness of the dielectric tube causes an increase in the accelerating gradient, and for $r_{2}=150 \mu \mathrm{m}$ it is $888.5 \mathrm{MeV} / \mathrm{m}$. Though such an acceleration gradient is less than optimum, it is high enough to recommend a structure with a thick inner tube as a possible candidate for a prototype of the wakefield accelerator. An increase in the outer radius of the inner shell causes the location of the first maximum of a

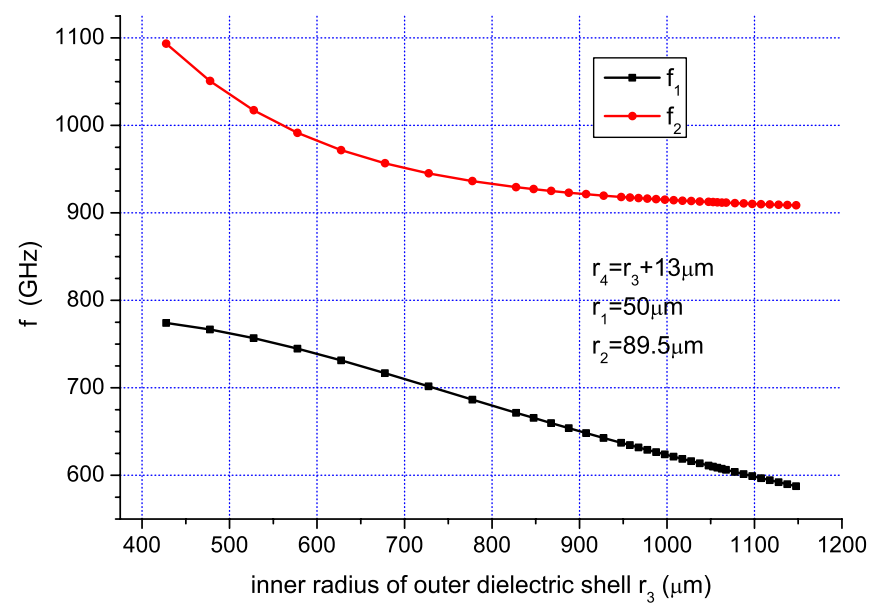

FIG. 8. (Color) Dependence of the first two frequencies of excited modes upon the width of the drive channel. The thickness of the outer dielectric shell is fixed and equal to $13 \mu \mathrm{m}$. The other parameters are as in Table I. 


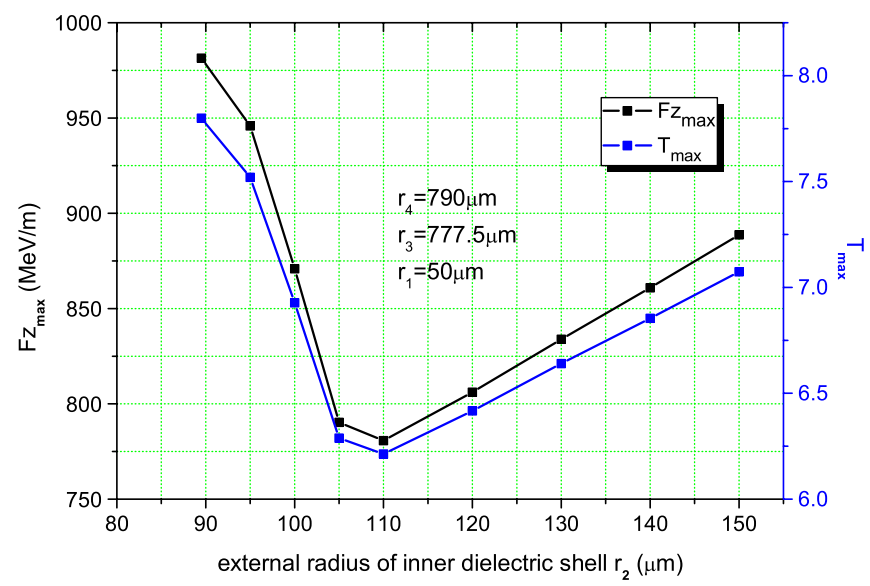

FIG. 9. (Color) The value of the composite longitudinal electric field at the first axial maximum and corresponding transformer ratio versus the external radius of the inner dielectric shell. The radii of the outer dielectric shell are $r_{3}=777.5 \mu \mathrm{m}$ and $r_{4}=$ $790.5 \mu \mathrm{m}$. The rest of the parameters are the same as in Table I.

field to change nonmonotonically (see Fig. 10). This is caused by the complex interference of some of the radial modes that form the composite field.

Having fixed the position and a thickness of the inner dielectric cylinder, we can investigate the dependence of the accelerating field on the thickness of the outer dielectric shell. Let the radii of the inner shell be taken as 50 and $150 \mu \mathrm{m}$. In Figs. 11 and 12, dependencies of the first maximum of accelerating field and its axial location and transformer ratio, upon the inner radius of the outer dielectric shell $r_{3}$, are given. Dependence of the accelerating field on the thickness of the outer dielectric shell has two local maxima. The first is near the initial shell thickness of $13 \mu \mathrm{m}\left(r_{3}=777.5 \mu \mathrm{m}\right)$ and the second is when shell thickness is approximately $83 \mu \mathrm{m}\left(r_{3}=707.5 \mu \mathrm{m}\right)$. The

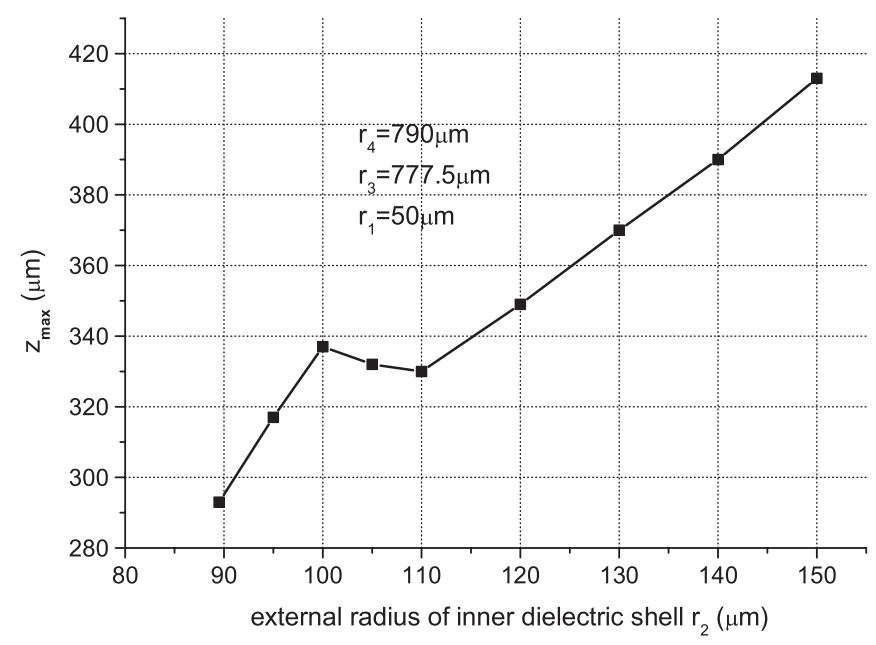

FIG. 10. The location (from drive bunch center) of the first maximum of the accelerating field versus width of the drive channel. The parameters are the same as those in Fig. 9

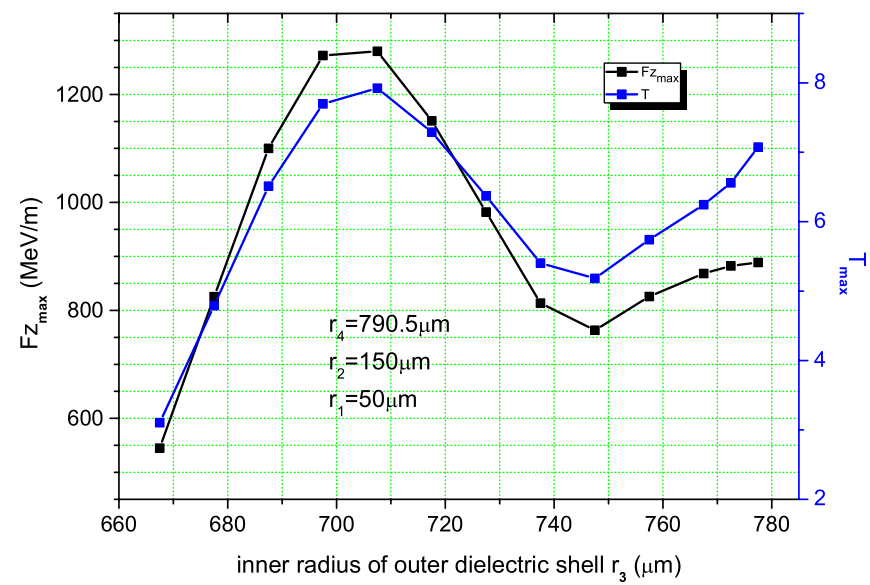

FIG. 11. (Color) The value of the composite longitudinal electric field at the first axial maximum and corresponding transformer ratio versus the inner radius of the outer dielectric shell. The radii of the inner dielectric shell are 50 and $150 \mu \mathrm{m}$. The external radius of the outer dielectric shell is $790 \mu \mathrm{m}$. The other parameters are the same as those in Table I.

amplitude of the field at the second maximum exceeds the amplitude at the first maximum and is equal $1.28 \mathrm{GeV} / \mathrm{m}$, located at a distance of $689 \mu \mathrm{m}$ from the center of the drive bunch. The transformer ratio at the second maximum is 7.92. The dependence of the transformer ratio qualitatively follows the dependence of the accelerating field maximum behavior. The axial distance from the center of the drive bunch to the field maximum increases on the average when we increase the thickness of the outer dielectric shell.

When increasing the thicknesses of the dielectric shells, the number of modes giving an important contribution to the composite field increases. As an example, in Fig. 13 are shown the radial profiles of $E_{0 m}$ mode amplitudes of lon-

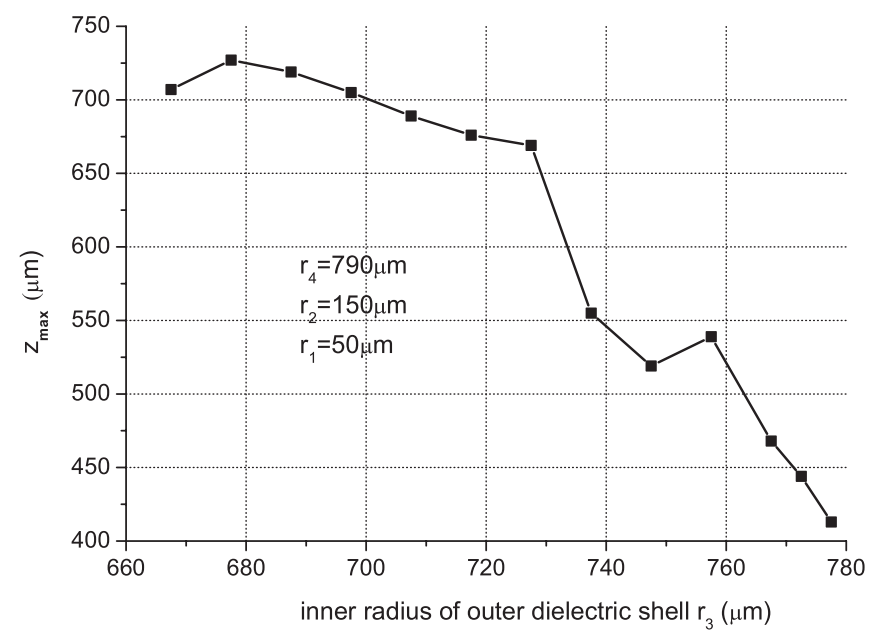

FIG. 12. The location (from drive bunch center) of the first maximum of the accelerating field versus the thickness of the inner radius of the outer dielectric shell. The parameters are the same as those in Fig. 11. 


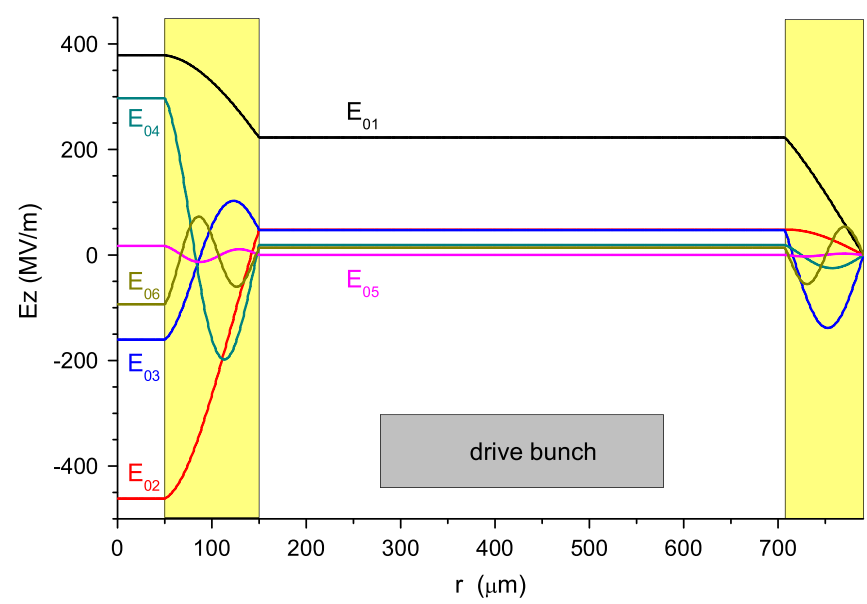

FIG. 13. (Color) Transverse profiles $E_{z}(r)$ for the first six TM modes. Radii of the inner dielectric shell are 50 and $150 \mu \mathrm{m}$, radii of the outer dielectric shell are 707.5 and $790.5 \mu \mathrm{m}$. Locations of the two dielectric shells are highlighted in yellow, and location and width of the 5-GeV drive bunch is shown in gray.

gitudinal electric field, and, in Fig. 14, axial profiles of the composite longitudinal force and its components which act upon a test electron at the axis of the accelerating channel in a coaxial dielectric waveguide having radii of $r_{1}=$ $50 \mu \mathrm{m}, \quad r_{2}=150 \mu \mathrm{m}, \quad r_{3}=707.5 \mu \mathrm{m}, \quad$ and $r_{4}=$ $790.5 \mu \mathrm{m}$. Six modes give significant contributions to the composite field excited by the electron bunch. Comparing Figs. 13 and 14 with similar, but considerably smaller, thicknesses of dielectric shells (Figs. 3 and 4) allows one to come to the conclusion that the increase in the thicknesses of the dielectric shells leads to enrichment of the spectrum of excited modes. As one might expect, as the frequencies of excited modes decrease, the longitudinal

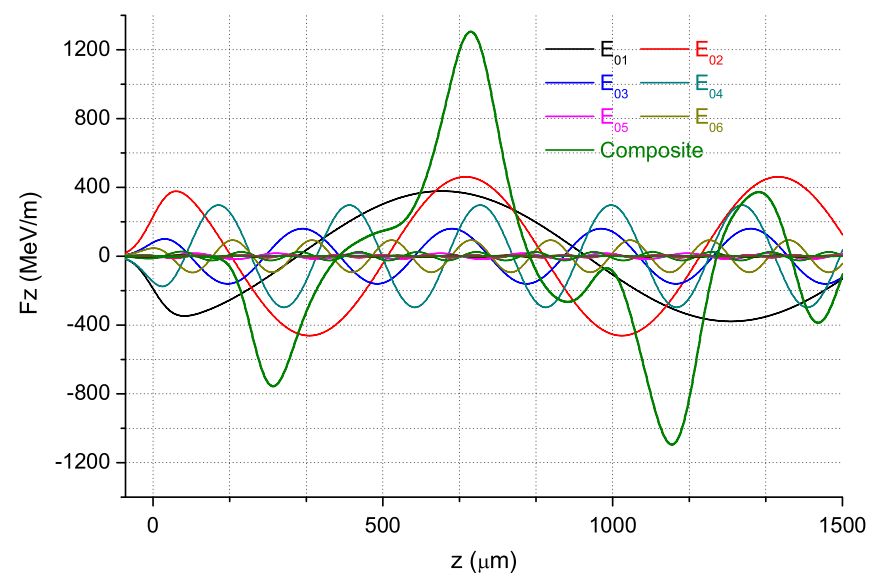

FIG. 14. (Color) Axial profiles of the longitudinal composite force and components of the force of the $E_{0 m}$ modes acting on a test electron along the center of the accelerating channel. The drive bunch moves from right to left and its center is located at $z=0$. The dimensions of the dielectric shell and parameters of the drive bunch are the same as those in Fig. 13. distance from the center of a drive bunch to the first maximum increases. The frequencies of the first six resonant waves in Figs. 13 and 14 in ascending order are $238.491 \mathrm{GHz}\left(E_{01}\right), 440.861 \mathrm{GHz}\left(E_{02}\right), 922.488 \mathrm{GHz}$ $\left(E_{03}\right), 1052.853 \mathrm{GHz}\left(E_{04}\right), 1677.309 \mathrm{GHz}\left(E_{05}\right)$, and $1733.222 \mathrm{GHz}\left(E_{06}\right)$.

\section{PIC SIMULATIONS}

In this section we show results from numerical simulations of excitation by an annular electron bunch of a coaxial dielectric structure, for which analytical studies were carried out in the previous sections. For this simulation we used the PIC solver of the CST PARTICLE STUDIO code of the CST STUDIO SUITE. A ring cathode with internal and external radii specified in Table I was placed at the input end of the structure as an electron source for the drive bunch. Its length has been chosen to be $10 \mu \mathrm{m}$, and the cathode surface is a perfect electric conductor (PEC). As for the emission model, a Gaussian emission longitudinal profile with a fixed electron energy of $5 \mathrm{GeV}$ and bunch charge of $6 \mathrm{nC}$ has been chosen. The rms length of the drive bunch was taken to be the same as for the analytical investigations in the previous section, i.e. $34.64 \mu \mathrm{m}$, and the cutoff length of the bunch has been chosen to be $2.5 \mathrm{rms}$ lengths of the bunch, i.e., it is equal to $86.6 \mu \mathrm{m}$. For boundary conditions we take the vanishing of the tangential component of electric field at all boundary surfaces, except for the output end of the structure where we applied an open boundary condition. For the best matching with free space, a vacuum gap of $50 \mu \mathrm{m}$ was added onto the dielectric structure.

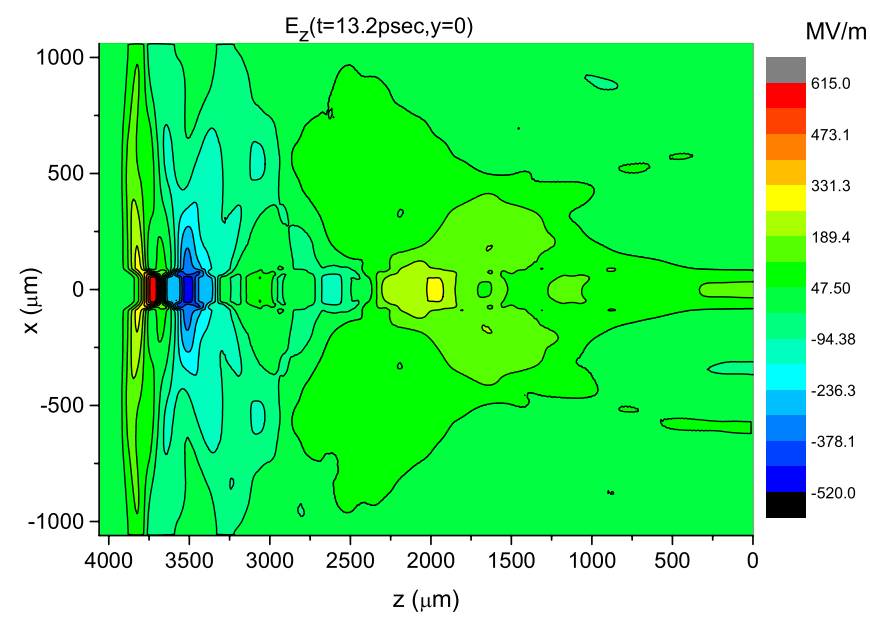

FIG. 15. (Color) Map of the axial wakefield in the coaxial DWFA. The origin of the Cartesian coordinate system is at the axis $(x=0, y=0)$, at the input end $(z=0)$ of the structure. The annular bunch drives these fields, and its center is located on the far left from the entrance into DWFA at the $z=3880 \mu \mathrm{m}$. The wakefield pattern moves with the drive bunch, towards the left, and it has been followed here for $t=13.2 \mathrm{psec}$. 
In Fig. 15, the image/contour plot of axial electric field in the $x-z$ plane $(y=0)$ of the coaxial structure for time $t=13.2 \mathrm{psec}$ is shown. The bunch head is approximately at a distance of $3967 \mu \mathrm{m}$ from the entrance to the structure. One can see the Cherenkov cones of the radiation at the forward front of wakefields in the inner dielectric tube. The wakefield in the accelerating channel at short distances from the drive bunch is very strong. With increasing distance behind the drive bunch the field energized in the structure becomes more and more irregular. This irregular character of the wakefield is caused by interference between Cherenkov radiation, the quenching wave, and transition radiation [4]. The quenching wave interferes with the field of the Cherenkov wakefield radiation behind the wave group velocity front of the Cherenkov radiation. When we use thin dielectric shells, the group velocity is close to the bunch velocity and therefore the excited field has considerable amplitude only for small distances behind the drive bunch. This conclusion is confirmed by results of the numerical computations presented in Figs. 15 and 16. Behind the drive bunch there is only one strongly pronounced maximum of the accelerating field. The accelerated bunch would be positioned at the center of the intense blue stripe at $z=3500 \mu \mathrm{m}$.

Sections of the image/contour plot of the electric field are presented in Fig. 16 where one-dimensional profiles of electric field along the centers of the accelerating and the drive channels are presented. The accelerating field has a maximum of $520 \mathrm{MV} / \mathrm{m}$, located at $z=3500 \mu \mathrm{m}$, i.e., in longitudinal direction it is displaced $380 \mu \mathrm{m}$ from the center of the drive bunch. The position and the value of the first maximum of the accelerating field show good agreement between the analytical results of the previous section and the results of the particle-in-cell simulation. However, as Figs. 15 and 16 show, the validity of the analytical results presented in the previous section is ap-

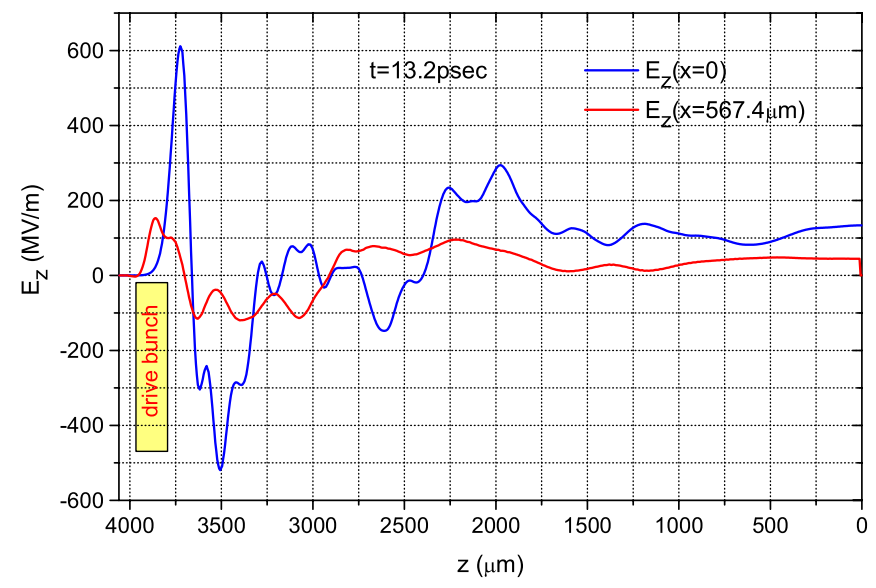

FIG. 16. (Color) Axial wakefields at the centers of drive (red line) and accelerating channels (blue line) of the coaxial DWFA for time $t=13.2 \mathrm{psec}$ when the drive bunch center is located at $z=3880 \mu \mathrm{m}$ from the entrance from the structure $(z=0)$.

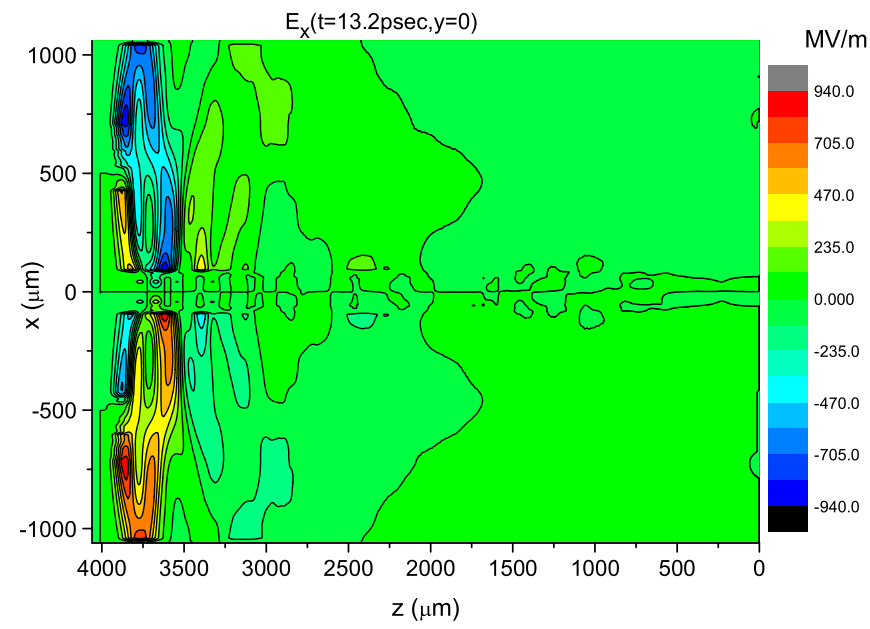

FIG. 17. (Color) Map of the transverse component of electric field along the structure for time $t=13.2 \mathrm{psec}$. The annular drive bunch is at the left side of the map.

proximately limited here to a region extending from the drive bunch to the first maximum of the accelerating field. As the longitudinal electric field in the transverse section of the drive bunch is nearly homogeneous, from the data of Fig. 16 it is possible to calculate a transformer ratio $T \sim 6$.

The image/contour plot of the transverse electric field is shown in Fig. 17; in the transverse direction this field vanishes at the structure axis and inside the drive bunch, but it has a maximum at the bunch surface (from the space charge field) and at the inner surfaces of the dielectric shells. The maximum of the transverse field intensity is $\sim 880 \mathrm{MV} / \mathrm{m}$.

One important feature found by the PIC simulation is that in the vicinity of the accelerating field maximum the transverse force is focusing (see Fig. 18): the transverse force is negative while the displacement from the axis is

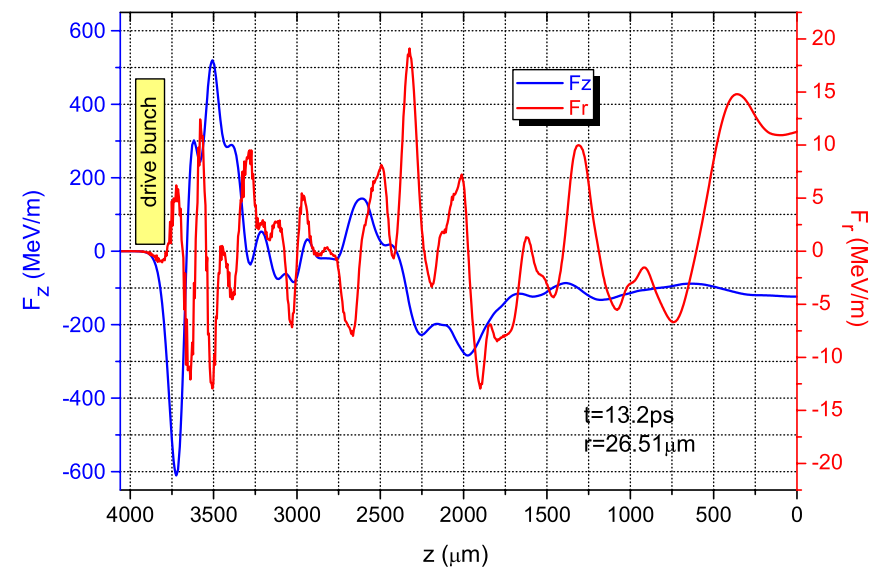

FIG. 18. (Color) Axial (blue line) and transverse (red line) forces acting upon electron versus $z$, at $r=26.51 \mu \mathrm{m}$ from the central axis in a witness bunch channel. The witness bunch would be placed at $z=3500 \mu \mathrm{m}$. 


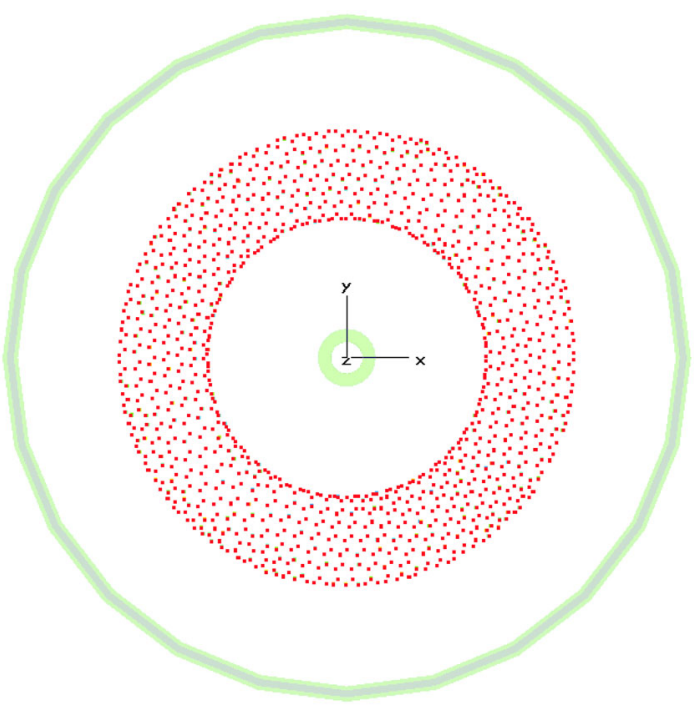

FIG. 19. (Color) The front view of drive bunch electrons after $13.2 \mathrm{psec}$ of the travel (bunch head is located at $3.967 \mathrm{~mm}$ from the structure input). Different colors of simulated electrons indicate different energy losses. Dielectric tubes are shown in green.

positive, thereby providing a stabilizing force for the radial motion of a witness particle. This is a distinct improvement in comparison with the case of the rectangular dielectric waveguide [15,18]. A restoring transverse force $\sim 12.5 \mathrm{MeV} / \mathrm{m}$ on a typical $5 \mathrm{GeV}$ witness electron at $z=$ $3500 \mu \mathrm{m}$ gives a betatron period of stable oscillation of $\sim 0.92 \mathrm{~m}$. Further studies have shown that the peak of this focusing transverse force tracks the motion of the longitudinal force accelerating peak as the drive bunch moves along the channel. Also, we find that it occurs even when the dimensions of the structure are altered in important ways. It appears to be caused by the quenching wave disturbance that originates at the input end of the structure. The second feature shown in the PIC simulation results is the strong influence of the quenching wave at distances beyond one period of the accelerating field, a result that is absent in the analytical formulation.

Though the electric and magnetic fields are strong on the surface of the drive bunch, the transverse force acting on the peripheral electrons is small because of their high relativistic factor. The maximum of the force acting on the drive bunch electrons is approximately the same as the force acting on the accelerated electrons. Therefore the transverse displacement of drive bunch particles will be insignificant. In Fig. 19, the front view (vertical projection onto the output end face plane) of the drive bunch electrons at the time when the bunch head is near the exit from the dielectric structure is shown. Notice that no particles have contacted the dielectric walls and the transverse profile of the bunch does not differ from the profile at the cathode.

We have also studied a six-zone structure where we use three annular vacuum channels. The inner one contains the witness bunch, the outer one contains the drive bunch, and the middle one is empty. This structure can provide a larger transformer ratio $(\sim 15)$ via a stepwise increase of longitudinal field that can occur at each dielectric shell.

\section{EXCITATION OF A TWO-CHANNEL COAXIAL STRUCTURE BY A BUNCH TRAIN}

In principle, a periodic sequence of electron bunches can be used to increase the accelerating field amplitude $[19,20]$. In a dielectric-lined structure operating in a single-mode excitation regime, the wakefields of a train of bunches spaced by the period of the wake wave are superimposed linearly. As a result, the excited field grows proportionally to the number of bunches. However, the negative influence of the quenching wave in longitudinally bounded dielectric structures restricts the number of bunches that can increase the overall wakefield amplitude by such superposition [4]. The greater the group velocity of wake wave, the more considerable is the interference by the quenching wave. When we decrease the thickness of the dielectric tubes or reduce their dielectric permittivity, the group velocity of the excited waves increases and the region of uncomplicated wakefield operation is narrowed. As shown in the previous section, this region of excitation of the dielectric structure by a single bunch can be as small as the distance to the first wakefield maximum. Therefore, in such a structure, using a sequence of drive bunches would not support a buildup of a larger accelerating field amplitude unless a much longer structure is provided.

We now study a different structure to demonstrate the possibility of coherent addition of wakefields in a coaxial two-channel accelerating structure that is to be excited by a train of periodically spaced bunches. The dielectric tubes in it are made from alumina, and this accelerating structure is designed for a frequency of the operating mode $\left(E_{02}\right.$ wave) of $\sim 30 \mathrm{GHz}$. The group velocity of the excited oscillations is less than the corresponding group velocity of waves in the $\mathrm{THz}$ structure investigated above. Therefore, the quenching disturbance lags further behind the leading drive bunch and so one can expect a coherent field addition of a short train of bunches; the structure and bunch parameters are listed in Table II.

In Fig. 20 we show the dependence of the longitudinal electric field in the accelerating channel axis versus distance from the center of the first bunch. The structure is energized by a sequence of four bunches. These results are calculated by using the analytical expressions of Sec. III, which do not include the quenching wave effects. The bunch spacing is taken to be the period of the $E_{02}$ mode, and as one would expect, the amplitude of the $E_{02}$ mode increases linearly with distance from the first bunch and its amplitude after the fourth bunch is greater by 4 times than amplitude of the $E_{02}$ mode after the first bunch. The composite field also grows from bunch to bunch. But, because of the considerable amplitude of the $E_{01}$ mode, 
TABLE II. Parameters for a GHz two-channel coaxial wakefield accelerator module using alumina dielectric tubes.

\begin{tabular}{lc}
\hline \hline Frequency of the $E_{02}$ design mode & $28.092 \mathrm{GHz}$ \\
External radius of outer coaxial dielectric shell $r_{4}$ & $14.05 \mathrm{~mm}$ \\
Inner radius of outer coaxial dielectric shell $r_{3}$ & $13.51 \mathrm{~mm}$ \\
External radius of inner coaxial dielectric shell $r_{2}$ & $3.18 \mathrm{~mm}$ \\
Accelerating channel radius (inner radius of inner coaxial dielectric shell) $r_{1}$ & $2.0 \mathrm{~mm}$ \\
Relative dielectric constant of dielectric shells $\varepsilon$ & 9.8 \\
rms bunch length $\sigma_{z}$ (Gaussian charge distribution) & $1 \mathrm{~mm}$ \\
Outer drive bunch radius (box charge distribution) $r_{b 2}$ & $10.39 \mathrm{~mm}$ \\
Inner drive bunch radius $r_{b 1}$ & $6.39 \mathrm{~mm}$ \\
Bunch energy & $14 \mathrm{MeV}$ \\
Bunch charge & $50 \mathrm{nC}$ \\
\hline \hline
\end{tabular}

the rate of rise of the composite field is less than linear. After the fourth bunch the accelerating gradient is about $50 \mathrm{MeV} / \mathrm{m}$; use of a single bunch results in a maximum value of accelerating gradient of $\sim 19 \mathrm{MeV} / \mathrm{m}$.

For comparison with these analytical results, in Fig. 21 we show the results of a PIC simulation of the excitation of this coaxial dielectric structure by the same bunch train. From the head of the bunch train, the accelerating gradient amplitude grows almost linearly as we move away from the wakefield front. Just after the last (fourth) drive bunch, the gradient reaches a maximum of $47.4 \mathrm{MeV} / \mathrm{m}$, and then begins to decrease because of destructive interference between the wakefield and the slower-moving quenching wave disturbance. The accelerating gradient from a single bunch at the first field maximum is $16.3 \mathrm{MeV} / \mathrm{m}$. Thus, there is a good correspondence between the analytical calculation and the PIC simulation for the value of the

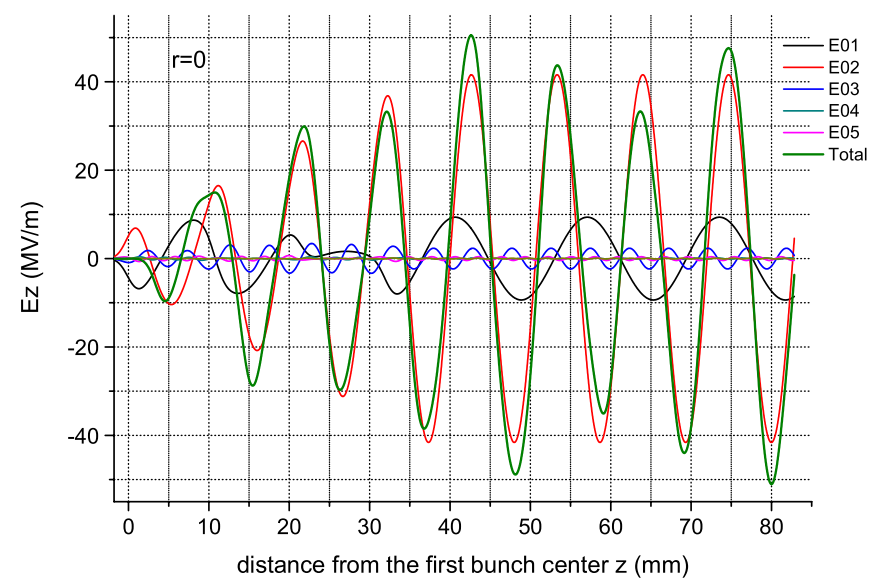

FIG. 20. (Color) Results of analytical computations of axial profiles of the longitudinal composite field (green line) and longitudinal fields of the $E_{0 m}$ modes (labeled as $E_{0 m}$ ) excited by the train of four bunches along the center of the accelerating channel. Drive bunches move from right to left and the center of the first bunch is located at $z=0$. Bunch spacing is about $10.7 \mathrm{~mm}$. accelerating gradient maximum. For the PIC simulation, there also is a nearly linear buildup of field amplitude behind the front edge of the wakefield. A likely explanation is that the $E_{01}$ wave has a greater group velocity than the $E_{02}$ mode and, consequently, it is not being enhanced much by the subsequent bunches.

In the previous section we showed from a PIC simulation of the excitation of a $\mathrm{THz}$ coaxial dielectric structure by a single bunch that the transverse force in the vicinity of an accelerating field maximum in the witness bunch channel is focusing (see Fig. 18). As seen in Fig. 21, this excellent property can also occur when the $\mathrm{GHz}$ structure is excited by a bunch train. At the maximum of accelerating field $(z=127.7 \mathrm{~mm})$, the value of the focusing force acting on a witness electron that is located at a distance of $0.98 \mathrm{~mm}$ from the axis is $1.68 \mathrm{MeV} / \mathrm{m}$. We have found in

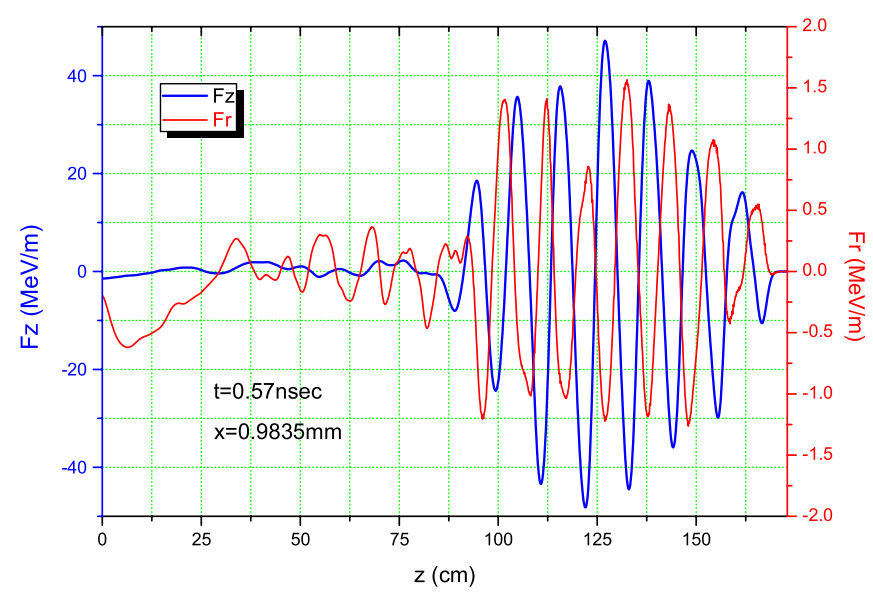

FIG. 21. (Color) Results of PIC simulations of axial (blue line) and transverse (red line) forces acting upon an electron versus $z$, at $r=0.98 \mathrm{~mm}$ from the central axis in a witness bunch channel. The coaxial structure is excited by the train of four bunches. Drive bunches move from left to right. The head of the first bunch is located at $z \sim 174 \mathrm{~mm}$. Bunch spacing is $10.7 \mathrm{~mm}$. The cathode length is $3 \mathrm{~mm}$, the length of dielectric tubes is $173 \mathrm{~mm}$, and the length of the extra vacuum gap is $10 \mathrm{~mm}$. 
subsequent simulations that it is possible to adjust the difference of phase between the longitudinal and transverse forces at a maximum of an accelerating field by making a small change of the bunch-repetition period.

The transverse forces acting on the electrons of the drive bunches (as well as for the case where the dielectric structure is excited by a single bunch) are insignificant and do not increase progressively from a leading bunch to a following bunch. For example, at the time $t=$ $0.57 \mathrm{nsec}$ the inner edge electrons (at $x=6.32 \mathrm{~mm}$ ) of the second drive bunch experience a maximum defocusing transverse force $\sim 0.44 \mathrm{MeV} / \mathrm{m}$, the third bunch $\sim 0.71 \mathrm{MeV} / \mathrm{m}$, and the fourth bunch $\sim 0.63 \mathrm{MeV} / \mathrm{m}$. At such values of deflecting forces the trailing drive bunch electrons should travel a longitudinal distance $\sim 60-70 \mathrm{~cm}$ before they begin to strike the walls of the inner dielectric tube. In Fig. 22, the front view of electrons in the fourth drive bunch near the output end of the dielectric structure is shown. It is seen that the cross-section location of the bunch electrons nearly replicates the cathode outline. Their transverse displacement is insignificant and these numerical simulations have found even less displacement than the linear estimates above. The investigation of the radial dynamics of all bunches has shown that the greatest radial displacement is suffered by electrons in the third bunch (although this is comparable with those of the last bunch). Such dependence proves also to be true for the longitudinal distribution of radial forces at the surfaces corresponding to the initial internal and external radii of a bunch. Though these particles are in a smaller braking field, the maximum of the defocusing force acting on these peripheral electrons is greater than the force acting on the particles of the fourth bunch.

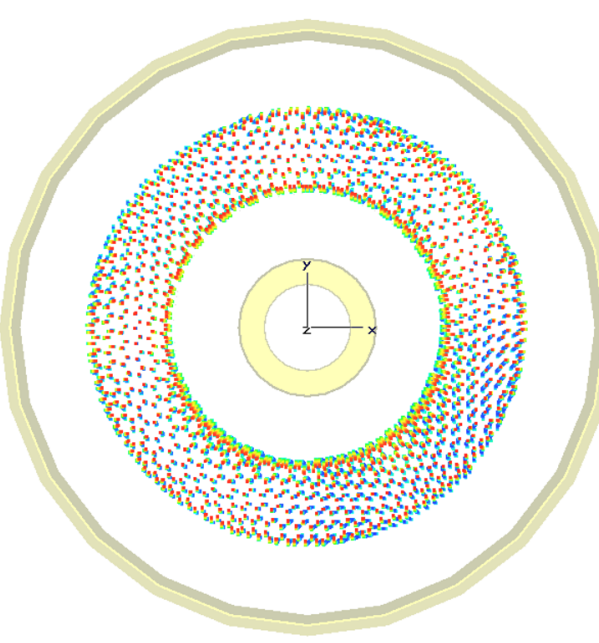

\section{$\mathrm{MeV}$}

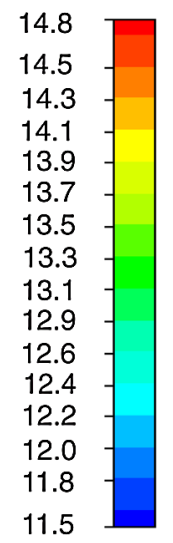

FIG. 22. (Color) Front view of the last bunch in a train of four bunches after $0.67 \mathrm{nsec}$ following injection of the first bunch. The head of this bunch is located at $170 \mathrm{~mm}$ from the structure input. Different colors of simulated electrons indicate different energy losses. Dielectric tubes are marked with yellow color.

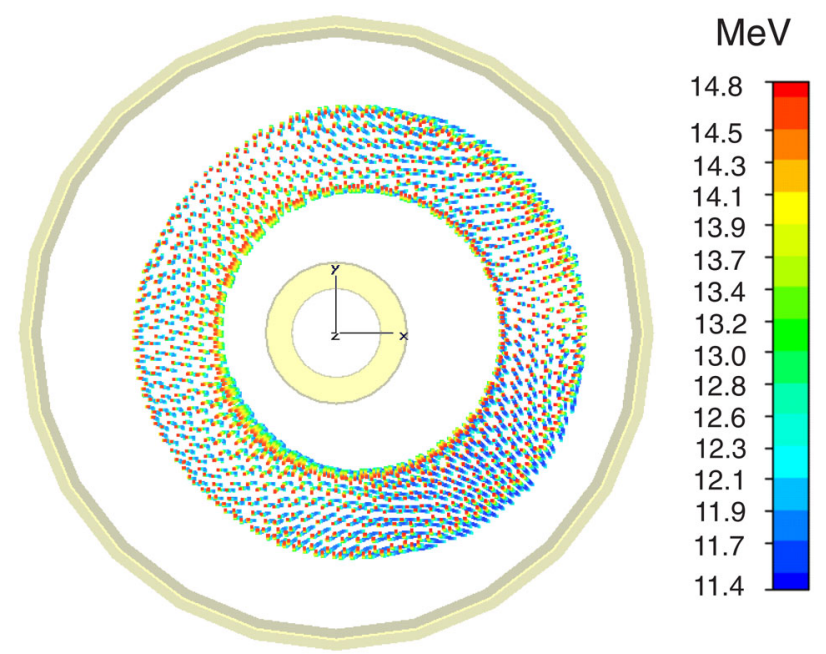

FIG. 23. (Color) The same case as in Fig. 22, but for bunches which are displaced initially by $1 \mathrm{~mm}$ to the right from the structure axis.

The off-centered drive bunch causes a deflecting force on the axis of the witness channel amounting to somewhat more than the focusing force, so in practice a somewhat smaller offset would be necessary to assure stable motion of the witness bunch.

Thus, the flat radial profile of the axial electric field $E_{z}(r)$ allows the bunch train to move stably for long distances. For additional study of the radial and azimuthal stability of the drive bunches, we investigated their dynamics when the initial position of their centers was displaced from the structure axis. In Fig. 23 the front view of particles of the fourth bunch is shown at the same time as for the case of the train of the centered bunches (Fig. 22). The offset of all four bunches is $1 \mathrm{~mm}$. As in the case of the train of the centered bunches, the radial displacement of particles of the last bunch is insignificant. Comparing the energy distributions of electrons in Figs. 22 and 23, it is possible to see a much more nonuniform distribution with azimuthal angle. If in the case of the centered bunches an azimuthal inhomogeneity develops owing to the discreteness of the initial distribution of macroparticles on a spatial grid, in the case of the off-centered bunches, the excitation of the higher of the azimuthal modes should be facilitated by the initial nonuniform distribution of a charge along the azimuth. But we find that in both cases, an energy modulation of particles has not led to a significant development of transverse instability of the bunches. Numerical study of the drive bunch motion is limited by computing power, and thus these results may not be applicable for bunch motion over a long distance.

A problem with using the multiple drive bunch train technique in general is that the drag forces increase on the trailing bunches, and this might complicate the design of a staged collider. In our example, the average drag force on the first drive bunch is $\sim 2.4 \mathrm{MeV} / \mathrm{m}$, whereas the drag on the fourth bunch is $\sim 7.1 \mathrm{MeV} / \mathrm{m}$. However, deploying a 
multiple drive bunch is a useful option if the charge available for a single drive bunch is limited for technical reasons. In that case the sum total of the charge in the multiple bunch train would be the same as that of the single bunch, and the aggregate drag would be approximately the same.

\section{DISCUSSION}

A coaxial, two-channel, dielectric-loaded cylindrical structure has been studied using analytic theory with numerical examples, as well as a numerical simulation using the PIC solver of the CST PARTICLE STUDIO code, for the purpose of gaining understanding of a possible new highgradient accelerator based on dielectric wakefield acceleration principles. The analytic theory takes the assumption of a structure that is infinitely extended along the axis, whereas the PIC simulation includes the effect of injection of charge bunches into a finite length of structure. The effects of the latter are important, because the quenching waves emitted by the necessary open input boundary condition move with a group velocity that is a large fraction of the speed of light in cases where the dielectric loading is slight, and thereby cause interference with the Cherenkov wakefields that trail the drive bunch with relativistic electron bunch speed. This interference is substantial because the quenching waves have amplitude comparable to the Cherenkov wakefields, as they must cancel the latter at the input boundary. Good agreement between the results of analytic theory and the PIC simulation has been obtained in the spatial region where both can apply.

A small, but not excessively small, structure has been examined for suitability as a linear accelerator of electrons (or positrons). Two-channel coaxial operation can achieve a gradient exceeding $1 \mathrm{GeV} / \mathrm{m}$ with a transformer ratio $\sim 8$, and possibly higher, in an optimized design, by using a single annular $6 \mathrm{nC}, 5 \mathrm{GeV}$ drive bunch to excite the structure. A trailing witness bunch in the second, central channel can thereby be accelerated stably to high energy using several modules that could be precisely fabricated and aligned. Radially uniform axial electric fields in the two vacuum channels permit a uniform drag (for the drive bunch) or acceleration (for the witness bunch) on all the particles in these bunches, and allow both types of bunches to move appreciable distances without distortion or wall contact. Stable transverse motion of the witness bunch particles should permit the preservation of bunch shape during acceleration without the use of external focusing structures. Use of a short train of drive bunches to enhance performance has been found to be practical when the quenching wave effects can be offset by choice of design parameters. A cathode structure that emits a ring-type bunch of charge has been studied in the past, in connection with a wakefield transformer experiment [21].

In the structure described in this paper, the lateral walls of the coaxial structure are lined with a suitable dielectric that has demonstrated ability to withstand high electric stress and resist wall surface charge buildup. (We have found that the dielectric need not be the same for each cylindrical wall.) A DWFA having microwave-scale dimensions that uses drive bunches of several MeV energy might support the inner dielectric cylinder with a low- $Z$ metallic foil, through which the annular drive bunch can pass with negligible energy loss or structural damage to the foil. In that case, a wall thickness $\sim 200 \mu \mathrm{m}$ of aluminum might be suitable. (We point out that the entry boundary condition we used for the PIC simulation was a metallic one, namely, zero radial and azimuthal electric fields at $z=0$ ). For a small high-gradient structure having millimeter-scale transverse dimensions, the supporting "foil" might be a carbon nanotube fabric having thickness $\sim 25 \mu \mathrm{m}$ and good electrical conductivity. Support of the inner dielectric tube inside a lengthy accelerator module might require the periodic use of an adjustable supporting carbon nanotube filament. Answers to questions regarding the utility and durability of such materials and the techniques to maintain precise alignment in a coaxial DWFA, as also the matter of production of an annular drive bunch with adequate charge, require a significant research and development effort and are beyond the scope of this study.

We compare the results obtained here with our study of the two-channel rectangular DWFA [15]. The cylindrical and rectangular configurations share many common advantages, such as the separated bunch lines, the enhanced transformer ratio, and the continuous coupling of energy from the drive bunch to the witness bunch. However, in order to obtain useful gradients, the rectangular structures cannot be tall, and therefore it suffers from significant deflecting forces that act upon both the drive bunches and the witness bunches. Furthermore, the accelerating gradients are smaller for rectangular units than for the cylindrical ones, the latter benefiting from the $r=0$ point of symmetry. While it is possible to position a witness bunch at an axial position behind the drive bunch where the axial gradient is large and the transverse force is comparatively small in the rectangular unit [15], nevertheless these transverse forces do vary along the length of the witness bunch and this will result in bunch distortion as the bunch traverses the accelerating module. In contrast, the cylindrical structure provides a stabilizing transverse force for the witness bunch, a gift, surprisingly enough, of the quenching waves.

Recently [22], analysis was published for wakefields on a four-zone coaxial structure energized by an infinitesimally thin ring drive bunch, without consideration of the quenching wave.

\section{ACKNOWLEDGMENTS}

This research was supported by U.S. Department of Energy, Office of High Energy Physics, Advanced Accelerator R\&D. 
[1] C. Wang and J. L. Hirshfield, Phys. Rev. ST Accel. Beams 9, 031301 (2006).

[2] S. Y. Park and J.L. Hirshfield, Phys. Rev. E 62, 1266 (2000).

[3] I. N. Onischenko, D. Yu. Sidorenko, and G. V. Sotnikov, Phys. Rev. E 65, 066501 (2002).

[4] T. C. Marshall, N. I. Onischenko, and G. V. Sotnikov, in Advanced Accelerator Concepts: 11th Workshop, edited by Vitaly Yakimenko, AIP Conf. Proc. No. 737 (AIP, New York, 2004), pp. 698-707.

[5] A. Sessler and G. Westenskow, in Handbook of Accelerator Physics and Engineering, edited by A.W. Chao and M. Tigner (World Scientific, New Jersey, 2002), pp. 46-48.

[6] J.P. Delahaye, "The Compact Linear Collider (CLIC) Study," CERN Particle Physics Seminar, http://clic-study. web.cern.ch/CLIC-Study/Presentations/20050421.pdf.

[7] E. Chojnacki, W. Gai, P. Schoessow, and J. Simpson, in Proceedings of the IEEE 1991 Particle Accelerator Conference (APS Beams Physics) (IEEE, Piscataway NJ, 1991), Vol. 5, pp. 2557-2559.

[8] M. E. Conde, W. Gai, R. Konecny, J. Power, P. Schoessow, and P. Zou, in Advanced Accelerator Concepts: Eighth Workshop, edited by W. Lawson, C. Bellamy, and D. F. Brosius, AIP Conf. Proc. No. 472 (AIP, New York, 1998), pp. 626-634.

[9] C. Jing, W. M. Liu, W. Gai, J. G. Power, and T. Wong, Nucl. Instrum. Methods Phys. Res., Sect. A 539, 445 (2005).

[10] L. Schachter, R. L. Byer, and R. H. Siemann, Phys. Rev. E 68, 036502 (2003).

[11] A. M. Altmark and A. D. Kanareykin, Tech. Phys. Lett. 34, 174 (2008).

[12] M.C. Thompson, H. Badakov, A. M. Cook, J. B. Rosenzweig, R. Tikhoplav, G. Travish, I. Blumenfeld, M. J. Hogan, R. Ischebeck, N. Kirby, R. Siemann, D. Walz, P. Muggli, A. Scott, and R. B. Yoder, Phys. Rev. Lett. 100, 214801 (2008).

[13] We observe that formerly a matrix approach was applied to derive the dispersion equation of a stratified Bragg fiber [14]. The transfer matrices determined there connect fields in two adjacent layers at the same boundary, on the left and to the right of it. The definition of transition matrices used in Ref. [1] allows one to derive a much more compact form of the dispersion equation.

[14] P. Yeh, A. Yariv, and E. Marom, J. Opt. Soc. Am. 68, 1196 (1978).

[15] G. V. Sotnikov, I. N. Onishchenko, J.L. Hirshfield, and T. C. Marshall, Probl. At. Sci. Technol., Ser. Nucl. Phys. Investigations 49, 148 (2008).

[16] T. C. Marshall, G. V. Sotnikov, S. V. Shchelkunov, and J. L. Hirshfield, in Advanced Accelerator Concepts: 13th Workshop, edited by Carl B. Schroeder, Wim Leemans, and Eric Esarey, AIP Conf. Proc. No. 1086 (AIP, New York, 2009), pp. 421-426.

[17] Hereafter we will analyze (for the reasons which will be clear from following section) only the first maximum of an accelerating field.

[18] G. V. Sotnikov, T. C. Marshall, S. V. Shchelkunov, A. Didenko, and J.L. Hirshfield, in Advanced Accelerator Concepts: 13th Workshop (Ref. [16]), pp. 415-420.

[19] I. N. Onishchenko, V. A. Kiseljob, A. K. Berezin, G. V. Sotnikov, V. V. Uskov, A. F. Linnik, and Ya. B. Fainberg, in Proceedings of the 1995 Particle Accelerator Conference (IEEE, New York, 1995), pp. 782-783.

[20] C. Jing, A. Kanareykin, J. G. Power, M. Conde, Z. Yusof, P. Schoessow, and W. Gai, Phys. Rev. Lett. 98, 144801 (2007).

[21] M. Bieler, W. Bialowons, H.-D. Bremer, F.-J. Decker, H.-Ch. Lewin, P. Schütt, G.-A. Voss, R. Wanzenberg, and T. Weiland, http://accelconf.web.cern.ch/AccelConf/ e88/PDF/EPAC1988_0967.PDF; also, F.-J. Decker, W. Bialowons, M. Bieler, H.-D. Bremer, H.-C. Lewin, P. Schütt, G.-A. Voss, R. Wanzenberg, and T. Weiland, http://accelconf.web.cern.ch/AccelConf/e88/PDF/ EPAC1988_0613.PDF.

[22] Wanming Liu and Wei Gai, Phys. Rev. ST Accel. Beams 12, 051301 (2009). 